\title{
On resonances and bound states of Smilansky Hamiltonian
}

\author{
P. Exner, V. Lotoreichik, M. Tater \\ Nuclear Physics Institute, Czech Academy of Sciences, 25068 Řež, Czech Republic \\ exner@ujf.cas.cz, lotoreichik@ujf.cas.cz, tater@ujf.cas.cz
}

PACS 02.30.Tb, 03.65.Db

DOI 10.17586/2220-8054-2016-7-5-789-802

We consider the self-adjoint Smilansky Hamiltonian $\mathbf{H}_{\varepsilon}$ in $L^{2}\left(\mathbb{R}^{2}\right)$ associated with the formal differential expression $-\partial_{x}^{2}-\frac{1}{2}\left(\partial_{y}^{2}+y^{2}\right)-$ $\sqrt{2} \varepsilon y \delta(x)$ in the sub-critical regime, $\varepsilon \in(0,1)$. We demonstrate the existence of resonances for $\mathrm{H}_{\varepsilon}$ on a countable subfamily of sheets of the underlying Riemann surface whose distance from the physical sheet is finite. On such sheets, we find resonance free regions and characterize resonances for small $\varepsilon>0$. In addition, we refine the previously known results on the bound states of $H_{\varepsilon}$ in the weak coupling regime $(\varepsilon \rightarrow 0+)$. In the proofs we use Birman-Schwinger principle for $\mathrm{H}_{\varepsilon}$, elements of spectral theory for Jacobi matrices, and the analytic implicit function theorem.

Keywords: Smilansky Hamiltonian, resonances, resonance free region, weak coupling asymptotics, Riemann surface, bound states.

Received: 1 July 2016. Revised: 28 July 2016.

In memory of B. S. Pavlov (1936-2016)

\section{Introduction}

In this paper we investigate resonances and bound states of the self-adjoint Hamiltonian $\mathrm{H}_{\varepsilon}$ acting in the Hilbert space $L^{2}\left(\mathbb{R}^{2}\right)$ and corresponding to the formal differential expression

$$
-\partial_{x}^{2}-\frac{1}{2}\left(\partial_{y}^{2}+y^{2}\right)-\sqrt{2} \varepsilon y \delta(x) \quad \text { on } \mathbb{R}^{2},
$$

in the sub-critical regime, $\varepsilon \in(0,1)$. The operator $\mathrm{H}_{\varepsilon}$ will be rigorously introduced in Section 1.1 below. Operators of this type were suggested by U. Smilansky in [1] as a model of irreversible quantum system. His aim was to demonstrate that the 'heat bath' need not have an infinite number of degrees of freedom. On a physical level of rigor he showed that the spectrum undergoes an abrupt transition at the critical value $\varepsilon=1$. A mathematically precise spectral analysis of these operators and their generalizations has been performed by M. Solomyak and his collaborators in [2-8]. Time-dependent Schrödinger equation generated by Smilansky-type Hamiltonian is considered in [9].

By now many of the spectral properties of $\mathrm{H}_{\varepsilon}$ are understood. On the other hand, little attention has been paid so far to the fact that such a system can also exhibit resonances. The main aim of this paper is to initiate investigation of these resonances starting from demonstration of their existence. One of the key difficulties is that this model belongs to a class wherein the resolvent extends to a Riemann surface having uncountably many sheets. The same complication appears e.g. in studying resonances for quantum waveguides [10-13], [14, §3.4.2] and for general manifolds with cylindrical ends $[15,16]$.

In this paper, we prove the existence and obtain a characterization of resonances of $\mathrm{H}_{\varepsilon}$ on a countable subfamily of sheets whose distance from the physical sheet is finite in the sense explained below. On any such sheet we characterize a region which is free of resonances. As $\varepsilon \rightarrow 0+$, the resonances on such sheets are localized in the vicinities of the thresholds $\nu_{n}=n+1 / 2, n \in \mathbb{N}$. We obtain a description of the subset of the thresholds in the vicinities of which a resonance exists for all sufficiently small $\varepsilon>0$ and derive asymptotic expansions of these resonances in the limit $\varepsilon \rightarrow 0+$. No attempt has been made here to define and study resonances on the sheets whose distance from the physical sheet is infinite.

As a byproduct, we obtain refined properties of the bound states of $\mathrm{H}_{\varepsilon}$ using similar methods as for resonances. More precisely, we obtain a lower bound on the first eigenvalue of $\mathrm{H}_{\varepsilon}$ and an asymptotic expansion of the weakly coupled bound state of $\mathrm{H}_{\varepsilon}$ in the limit $\varepsilon \rightarrow 0+$.

Methods developed in this paper can also be useful to tackle resonances for the analog of Smilansky model with regular potential which is suggested in [17] and further investigated in $[18,19]$. 


\section{Notations}

We use notations $\mathbb{N}:=\{1,2, \ldots\}$ and $\mathbb{N}_{0}:=\mathbb{N} \cup\{0\}$ for the sets of positive and natural integers, respectively. We denote the complex plane by $\mathbb{C}$ and define its commonly used sub-domains: $\mathbb{C}^{\times}:=\mathbb{C} \backslash\{0\}, \mathbb{C}_{ \pm}:=\{\lambda \in$ $\mathbb{C}: \pm \operatorname{Im} \lambda>0\}$ and $\mathbb{D}_{r}\left(\lambda_{0}\right):=\left\{\lambda \in \mathbb{C}:\left|\lambda-\lambda_{0}\right|<r\right\}, \mathbb{D}_{r}^{\times}\left(\lambda_{0}\right):=\left\{\lambda \in \mathbb{C}: 0<\left|\lambda-\lambda_{0}\right|<r\right\}, \mathbb{D}_{r}:=\mathbb{D}_{r}(0)$, $\mathbb{D}_{r}^{\times}:=\mathbb{D}_{r}^{\times}(0)$ with $r>0$. The principal value of the argument for $\lambda \in \mathbb{C}^{\times}$is denoted by $\arg \lambda \in(-\pi, \pi]$. The branches of the square root are defined by:

$$
\mathbb{C}^{\times} \ni \lambda \mapsto(\lambda)_{j}^{1 / 2}:=|\lambda|^{1 / 2} e^{\mathrm{i}((1 / 2) \arg \lambda+j \pi)}, \quad j=0,1 .
$$

If the branch of the square root is not explicitly specified, we understand the branch $(\cdot)_{0}^{1 / 2}$ by default. We also set $\mathbf{0}=(0,0) \in \mathbb{C}^{2}$.

The $L^{2}$-space over $\mathbb{R}^{d}, d=1,2$, with the usual inner product is denoted by $\left(L^{2}\left(\mathbb{R}^{d}\right),(\cdot, \cdot)_{\mathbb{R}^{d}}\right)$ and the $L^{2}$-based first order Sobolev space by $H^{1}\left(\mathbb{R}^{d}\right)$, respectively. The space of square-summable sequences of vectors in a Hilbert space $\mathcal{G}$ is denoted by $\ell^{2}\left(\mathbb{N}_{0} ; \mathcal{G}\right)$. In the case that $\mathcal{G}=\mathbb{C}$ we simply write $\ell^{2}\left(\mathbb{N}_{0}\right)$ and denote by $(\cdot, \cdot)$ the usual inner product on it.

For $\xi=\left\{\xi_{n}\right\} \in \ell^{2}\left(\mathbb{N}_{0}\right)$, we adopt the convention that $\xi_{-1}=0$. Kronecker symbol is denoted by $\delta_{n m}$, $n, m \in \mathbb{N}_{0}$, we set $\mathrm{e}_{n}:=\left\{\delta_{n m}\right\}_{m \in \mathbb{N}_{0}} \in \ell^{2}\left(\mathbb{N}_{0}\right), n \in \mathbb{N}_{0}$, and adopt the convention that $\mathrm{e}_{-1}:=\{0\}$. We understand by $\operatorname{diag}\left(\left\{q_{n}\right\}\right)$ the diagonal matrix in $\ell^{2}\left(\mathbb{N}_{0}\right)$ with entries $\left\{q_{n}\right\}_{n \in \mathbb{N}_{0}}$ and by $\mathrm{J}\left(\left\{a_{n}\right\},\left\{b_{n}\right\}\right)$ the Jacobi matrix in $\ell^{2}\left(\mathbb{N}_{0}\right)$ with diagonal entries $\left\{a_{n}\right\}_{n \in \mathbb{N}_{0}}$ and off-diagonal entries $\left\{b_{n}\right\}_{n \in \mathbb{N}^{1}}$. We also set $\mathrm{J}_{0}:=\mathrm{J}(\{0\},\{1 / 2\})$.

By $\sigma(\mathrm{K})$, we denote the spectrum of a closed (not necessarily self-adjoint) operator $\mathrm{K}$ in a Hilbert space. An isolated eigenvalue $\lambda \in \mathbb{C}$ of $\mathrm{K}$ having finite algebraic multiplicity is a point of the discrete spectrum for $\mathrm{K}$; see $\left[23, \S\right.$ XII.2] for details. The set of all the points of the discrete spectrum for $\mathrm{K}$ is denoted by $\sigma_{\mathrm{d}}(\mathrm{K})$ and the essential spectrum of $\mathrm{K}$ is defined by $\sigma_{\mathrm{ess}}(\mathrm{K}):=\sigma(\mathrm{K}) \backslash \sigma_{\mathrm{d}}(\mathrm{K})$. For a self-adjoint operator $\mathrm{T}$ in a Hilbert space, we set $\lambda_{\text {ess }}(\mathrm{T}):=\inf \sigma_{\text {ess }}(\mathrm{T})$ and, for $k \in \mathbb{N}, \lambda_{k}(\mathrm{~T})$ denotes the $k$-th eigenvalue of $\mathrm{T}$ in the interval $\left(-\infty, \lambda_{\text {ess }}(\mathrm{T})\right)$. These eigenvalues are ordered non-decreasingly with multiplicities taken into account. The number of eigenvalues with multiplicities of the operator T lying in a closed, open, or half-open interval $\Delta \subset \mathbb{R}$ satisfying $\sigma_{\mathrm{ess}}(\mathrm{T}) \cap \Delta=\varnothing$ is denoted by $\mathcal{N}(\Delta ; \mathrm{T})$. For $\lambda \leq \lambda_{\text {ess }}(\mathrm{T})$ the counting function of $\mathrm{T}$ is defined by $\mathcal{N}_{\lambda}(\mathrm{T}):=\mathcal{N}((-\infty, \lambda) ; \mathrm{T})$.

\subsection{Smilansky Hamiltonian}

Define the Hermite functions:

$$
\chi_{n}(y):=e^{-y^{2} / 2} H_{n}(y), \quad n \in \mathbb{N}_{0} .
$$

Here, $H_{n}(y)$ is the Hermite polynomial of degree $n \in \mathbb{N}_{0}$ normalized by the condition $\left\|\chi_{n}\right\|_{\mathbb{R}}=1^{2}$. For more details on Hermite polynomials see [20, Chap. 22] and also [21, Chap. 5]. As it is well-known, the family $\left\{\chi_{n}\right\}_{n \in \mathbb{N}_{0}}$ constitutes an orthonormal basis of $L^{2}(\mathbb{R})$. Note also that the functions $\chi_{n}$ satisfy the three-term recurrence relation:

$$
\sqrt{n+1} \chi_{n+1}(y)-\sqrt{2} y \chi_{n}(y)+\sqrt{n} \chi_{n-1}(y)=0, \quad n \in \mathbb{N}_{0},
$$

where we adopt the convention $\chi_{-1} \equiv 0$. The relation (1.3) can be easily deduced from the recurrence relation [20, eq. 22.7.13] for Hermite polynomials. By a standard argument any function $U \in L^{2}\left(\mathbb{R}^{2}\right)$ admits unique expansion:

$$
U(x, y)=\sum_{n \in \mathbb{N}_{0}} u_{n}(x) \chi_{n}(y), \quad u_{n}(x):=\int_{\mathbb{R}} U(x, y) \chi_{n}(y) \mathrm{d} y,
$$

where $\left\{u_{n}\right\} \in \ell^{2}\left(\mathbb{N}_{0} ; L^{2}(\mathbb{R})\right)$. Following the presentation in [7], we identify the function $U \in L^{2}\left(\mathbb{R}^{2}\right)$ and the sequence $\left\{u_{n}\right\}$ and write $U \sim\left\{u_{n}\right\}$. This identification defines a natural unitary transform between the Hilbert spaces $L^{2}\left(\mathbb{R}^{2}\right)$ and $\mathcal{H}:=\ell^{2}\left(\mathbb{N}_{0} ; L^{2}(\mathbb{R})\right)$. For the sake of brevity, we denote the inner product on $\mathcal{H}$ by $\langle\cdot, \cdot\rangle$. Note that the Hilbert space $\mathcal{H}$ can also be viewed as the tensor product $\ell^{2}\left(\mathbb{N}_{0}\right) \otimes L^{2}(\mathbb{R})$.

For any $\varepsilon \in \mathbb{R}$, we define the subspace $\mathcal{D}_{\varepsilon}$ of $\mathcal{H}$ as follows: an element $U \sim\left\{u_{n}\right\} \in \mathcal{H}$ belongs to $\mathcal{D}_{\varepsilon}$ if, and only if

(i) $u_{n} \in H^{1}(\mathbb{R})$ for all $n \in \mathbb{N}_{0}$;

(ii) $\left\{-\left(u_{n,+}^{\prime \prime} \oplus u_{n,-}^{\prime \prime}\right)+\nu_{n} u_{n}\right\} \in \mathcal{H}$ with $u_{n, \pm}:=\left.u_{n}\right|_{\mathbb{R}_{ \pm}}$and $\nu_{n}=n+1 / 2$ for $n \in \mathbb{N}_{0}$;

\footnotetext{
${ }^{1}$ We do not distinguish between Jacobi matrices and operators in the Hilbert space $\ell^{2}\left(\mathbb{N}_{0}\right)$ induced by them, since in our considerations all the Jacobi matrices are bounded, closed, and everywhere defined in $\ell^{2}\left(\mathbb{N}_{0}\right)$.

${ }^{2}$ This normalization means that $H_{n}(y)$ is, in fact, a product of what is usually called the Hermite polynomial of degree $n \in \mathbb{N}_{0}$ with a normalization constant which depends on $n$.
} 
(iii) the boundary conditions

$$
u_{n}^{\prime}(0+)-u_{n}^{\prime}(0-)=\varepsilon\left(\sqrt{n+1} u_{n+1}(0)+\sqrt{n} u_{n-1}(0)\right)
$$

are satisfied for all $n \in \mathbb{N}_{0}$. For $n=0$ only the first term is present on the right-hand side.

By [7, Thm. 2.1], the operator:

$$
\operatorname{dom} \mathrm{H}_{\varepsilon}:=\mathcal{D}_{\varepsilon}, \quad \mathrm{H}_{\varepsilon}\left\{u_{n}\right\}:=\left\{-\left(u_{n,+}^{\prime \prime} \oplus u_{n,-}^{\prime \prime}\right)+\nu_{n} u_{n}\right\},
$$

is self-adjoint in $\mathcal{H}$. It corresponds to the formal differential expression (1.1). Further, we provide another way of defining $\mathrm{H}_{\varepsilon}$ which makes the correspondence between the operator $\mathrm{H}_{\varepsilon}$ and the formal differential expression (1.1) more transparent. To this aim, we define the straight line $\Sigma:=\left\{(0, y) \in \mathbb{R}^{2}: y \in \mathbb{R}\right\}$. Then, the Hamiltonain $\mathrm{H}_{\varepsilon}$, $\varepsilon \in(-1,1)$, can be alternatively introduced as the unique self-adjoint operator in $L^{2}\left(\mathbb{R}^{2}\right)$ associated via the first representation theorem [22, Thm. VI.2.1] with a closed, densely defined, symmetric, and semi-bounded quadratic form:

$$
\begin{aligned}
\mathfrak{h}_{\varepsilon}[u] & :=\left\|\partial_{x} u\right\|_{\mathbb{R}^{2}}^{2}+\frac{1}{2}\left\|\partial_{y} u\right\|_{\mathbb{R}^{2}}^{2}+\frac{1}{2}(y u, y u)_{\mathbb{R}^{2}}+\varepsilon \sqrt{2}\left(\left.\operatorname{sign}(y)|y|^{1 / 2} u\right|_{\Sigma},\left.|y|^{1 / 2} u\right|_{\Sigma}\right)_{\mathbb{R}}, \\
\operatorname{dom} \mathfrak{h}_{\varepsilon} & :=\left\{u \in H^{1}\left(\mathbb{R}^{2}\right): y u \in L^{2}\left(\mathbb{R}^{2}\right),|y|^{1 / 2}\left(\left.u\right|_{\Sigma}\right) \in L^{2}(\mathbb{R})\right\} .
\end{aligned}
$$

For more details and for the proof of equivalence between the two definitions of $H_{\varepsilon}$, see [7, $\left.\S 9\right]$. Since $H_{\varepsilon}$ commutes with the parity operator in $y$-variable, it is unitarily equivalent to $H_{-\varepsilon}$. We remark that the case $\varepsilon=0$ admits separation of variables. Thus, it suffices to study $\mathrm{H}_{\varepsilon}$ with $\varepsilon>0$.

In the following proposition, we collect fundamental spectral properties of $H_{\varepsilon}, \varepsilon \in(0,1)$, which are of importance in the present paper.

Proposition 1.1. Let the self-adjoint operator $\mathrm{H}_{\varepsilon}, \varepsilon \in(0,1)$, be as in (1.5). Then the following claims hold:

(i) $\sigma_{\mathrm{ess}}\left(\mathrm{H}_{\varepsilon}\right)=[1 / 2,+\infty)$;

(ii) $\inf \sigma\left(\mathrm{H}_{\varepsilon}\right) \geq \frac{1-\varepsilon}{2}$;

(iii) $1 \leq \mathcal{N}_{1 / 2}\left(\mathrm{H}_{\varepsilon}\right)<\infty$;

(iv) $\mathcal{N}_{1 / 2}\left(\mathrm{H}_{\varepsilon}\right)=1$ for all sufficiently small $\varepsilon>0$.

Items (i)-(iii) follow from [6, Lem 2.1] and [7, Thm. 3.1 (1),(2)]. Item (iv) is a consequence of [6, Thm. 3.2] and $[7, \S 10.1]$. Although we only deal with the sub-critical case, $\varepsilon \in(0,1)$, we remark that in the critical case, $\varepsilon=1$, the spectrum of $H_{1}$ equals to $[0,+\infty)$ and that in the sup-critical case, $\varepsilon>1$, the spectrum of $H_{\varepsilon}$ covers the whole real axis. Finally, we mention that in most of the existing literature on the subject not $\varepsilon>0$ itself but $\alpha=\sqrt{2} \varepsilon$ is chosen as the coupling parameter. We choose another normalization of the coupling parameter in order to simplify formulae in the proofs of the main results.

\subsection{Main results}

While we are primarily interested in the resonances, as indicated in the introduction, we have also a claim to make about the discrete spectrum which we present here as our first main result and which complements the results listed in Proposition 1.1.

Theorem 1.2. Let the self-adjoint operator $\mathrm{H}_{\varepsilon}, \varepsilon \in(0,1)$, be as in (1.5). Then the following claims hold.

(i) $\lambda_{1}\left(\mathrm{H}_{\varepsilon}\right) \geq 1-\sqrt{\frac{1}{4}+\varepsilon^{4}}$ for all $\varepsilon \in(0,1)$.

(ii) $\lambda_{1}\left(\mathrm{H}_{\varepsilon}\right)=\nu_{0}-\frac{\varepsilon^{4}}{16}+\mathcal{O}\left(\varepsilon^{5}\right)$ as $\varepsilon \rightarrow 0+$.

Theorem 1.2 (i) is proven by means of Birman-Schwinger principle. The bound in Theorem 1.2 (i) is non-trivial for $\varepsilon^{4}<3 / 4$. This bound is better than the one in Proposition 1.1 (ii) for small $\varepsilon>0$.

For the proof of Theorem 1.2 (ii) we combine Birman-Schwinger principle and the analytic implicit function theorem. We expect that the error term $\mathcal{O}\left(\varepsilon^{5}\right)$ in Theorem 1.2 (ii) can be replaced by $\mathcal{O}\left(\varepsilon^{6}\right)$ because the operator $\mathrm{H}_{\varepsilon}$ has the same spectral properties as $\mathrm{H}_{-\varepsilon}$ for any $\varepsilon \in(0,1)$. Therefore, the expansion of $\lambda_{1}\left(\mathrm{H}_{\varepsilon}\right)$ must be invariant with respect to interchange between $\varepsilon$ and $-\varepsilon$. In Lemma 4.1 given in Section 4 we derive an implicit scalar equation on $\lambda_{1}\left(\mathrm{H}_{\varepsilon}\right)$. This equation gives analyticity of $\varepsilon \mapsto \lambda_{1}\left(\mathrm{H}_{\varepsilon}\right)$ for small $\varepsilon$. It can also be used to compute higher order terms in the expansion of $\lambda_{1}\left(\mathrm{H}_{\varepsilon}\right)$. However, these computations might be quite tedious. 
Our second main result concerns the resonances of $\mathrm{H}_{\varepsilon}$. Before formulating it, we need to define the resonances rigorously. Let us consider the sequence of functions:

$$
r_{n}(\lambda):=\left(\nu_{n}-\lambda\right)^{1 / 2}, \quad n \in \mathbb{N}_{0} .
$$

Each of them has two branches $r_{n}(\lambda, l):=\left(\nu_{n}-\lambda\right)_{l}^{1 / 2}, l=0,1$. The vector-valued function $R(\lambda)=$ $\left(r_{0}(\lambda), r_{1}(\lambda), r_{2}(\lambda), \ldots\right)$ naturally defines the Riemann surface $\widehat{Z}$ with uncountably many sheets. With each sheet of $\widehat{Z}$ we associate the set $E \subset \mathbb{N}_{0}$ and the characteristic vector $l^{E}$ defined as:

$$
l^{E}:=\left\{l_{0}^{E}, l_{1}^{E}, l_{2}^{E}, \ldots\right\}, \quad l_{n}^{E}:= \begin{cases}0, & n \notin E \\ 1, & n \in E\end{cases}
$$

We adopt the convention that $l_{-1}^{E}=0$. The respective sheet of $\widehat{Z}$ is convenient to denote by $Z_{E}$. Each sheet $Z_{E}$ of $\widehat{Z}$ can be identified with the set $\mathbb{C} \backslash\left[\nu_{0},+\infty\right)$ and we denote by $Z_{E}^{ \pm}$the parts of $Z_{E}$ corresponding to $\mathbb{C}_{ \pm}$. With the notation settled, we define the realization of $R(\cdot)$ on $Z_{E}$ as:

$$
R_{E}(\lambda):=\left(r_{0}\left(\lambda, l_{0}^{E}\right), r_{1}\left(\lambda, l_{1}^{E}\right), r_{2}\left(\lambda, l_{2}^{E}\right), \ldots\right) .
$$

The sheets $Z_{E}$ and $Z_{F}$ are adjacent through the interval $\left(\nu_{n}, \nu_{n+1}\right) \subset \mathbb{R}, n \in \mathbb{N}_{0},\left(Z_{E} \sim_{n} Z_{F}\right)$, if their characteristic vectors $l^{E}$ and $l^{F}$ satisfy:

$$
\begin{aligned}
l_{k}^{F}=1-l_{k}^{E}, & \text { for } k=0,1,2, \ldots, n \\
l_{k}^{F}=l_{k}^{E}, & \text { for } k>n .
\end{aligned}
$$

We set $\nu_{-1}=-\infty$ and note that any sheet $Z_{E}$ is adjacent to itself through $\left(\nu_{-1}, \nu_{0}\right)$. In particular, the function $\lambda \mapsto R_{E}(\lambda)$ turns out to be componentwise analytic on the Riemann surface $\widehat{Z}$.

The sequence $\mathfrak{E}=\left\{E_{1}, E_{2}, \ldots, E_{N}\right\}$ of subsets of $\mathbb{N}_{0}$ is called a path if for any $k=1,2, \ldots, N-1$ the sheets $Z_{E_{k}}$ and $Z_{E_{k+1}}$ are adjacent. The following discrete metric:

$$
\rho(E, F):=\inf \left\{N \in \mathbb{N}_{0}: \mathfrak{E}=\left\{E_{1}, E_{2}, \ldots, E_{N}\right\}, E_{1}=E, E_{N}=F\right\},
$$

turns out to be convenient. The value $\rho(E, F)$ equals the number of sheets in the shortest path connecting $Z_{E}$ and $Z_{F}$. Note that for some sheets $Z_{E}$ and $Z_{F}$ a path between them does not exist and in this case we have $\rho(E, F)=\infty$. We identify the physical sheet with the sheet $Z_{\varnothing}$ (for $E=\varnothing$ ). A sheet $Z_{E}$ of $\widehat{Z}$ is adjacent to the physical sheet $Z_{\varnothing}$ if $\rho(E, \varnothing)=1$ and it can be characterised by existence of $N \in \mathbb{N}_{0}$ such that $l_{n}^{E}=1$ if, and only if $n \leq N$. Also, we define the component:

$$
\widetilde{Z}:=\cup_{E \in \mathcal{E}} Z_{E} \subset \widehat{Z}, \quad \mathcal{E}:=\left\{E \subset \mathbb{N}_{0}: \rho(E, \varnothing)<\infty\right\},
$$

of $\widehat{Z}$ which plays a distinguished role in our considerations. Any sheet in $\widetilde{Z}$ is located on a finite distance from the physical sheet with respect to the metric $\rho(\cdot, \cdot)$. The component $\widetilde{Z}$ of $\widehat{Z}$ in (1.11) can alternatively be characterized as:

$$
\widetilde{Z}=\cup_{F \in \mathcal{F}} Z_{F}, \quad \mathcal{F}:=\left\{F \subset \mathbb{N}_{0}: \sup \left\{n \in \mathbb{N}_{0}: l_{n}^{F}=1\right\}<\infty\right\} .
$$

The number of the sheets in $\widetilde{Z}$ is easily seen to be countable. In order to define the resonances of $\mathrm{H}_{\varepsilon}$ on $\widetilde{Z}$, we show that the resolvent of $\mathrm{H}_{\varepsilon}$ admits an extension to $\widetilde{Z}$ in a certain weak sense.

Proposition 1.3. For any $u \in L^{2}(\mathbb{R})$ and $n \in \mathbb{N}_{0}$ the function:

$$
\lambda \mapsto \mathfrak{r}_{n, \varepsilon}^{\varnothing}(\lambda ; u):=\left\langle\left(\mathrm{H}_{\varepsilon}-\lambda\right)^{-1} u \otimes \mathrm{e}_{n}, u \otimes \mathrm{e}_{n}\right\rangle
$$

admits unique meromorphic continuation $\mathfrak{r}_{n, \varepsilon}^{E}(\cdot ; u)$ from the physical sheet $Z_{\varnothing}$ to any sheet $Z_{E} \subset \widetilde{Z}$.

The proof of Proposition 1.3 is postponed until Appendix. Now we have all the tools to define resonances of $\mathrm{H}_{\varepsilon}$ on $\widetilde{Z}$.

Definition 1.4. Each resonance of $\mathrm{H}_{\varepsilon}$ on $Z_{E} \subset \widetilde{Z}$ is identified with a pole of $\mathfrak{r}_{n, \varepsilon}^{E}(\cdot ; u)$ for some $u \in L^{2}(\mathbb{R})$ and $n \in \mathbb{N}_{0}$. The set of all the resonances for $\mathrm{H}_{\varepsilon}$ on the sheet $Z_{E}$ is denoted by $\mathcal{R}_{E}(\varepsilon)$.

Our definition of resonances for $H_{\varepsilon}$ is consistent with [23, §XII.6], see also [14, Chap. 2] and [24] for multithreshold case. It should be emphasized that by the spectral theorem for self-adjoint operators the eigenvalues of $\mathrm{H}_{\varepsilon}$ are also regarded as resonances in the sense of Definition 1.4 lying on the physical sheet $Z_{\varnothing}$. This allows us to treat the eigenvalues and 'true' resonances on the same footing. Needless to say, bound states and true resonances correspond to different physical phenomena and their equivalence in this paper is merely a useful mathematical abstraction. 
According to Remark 2.5 below, the set of the resonances for $\mathrm{H}_{\varepsilon}$ on $Z_{E}$ is symmetric with respect to the real axis. Thus, it suffices to analyze resonances on $Z_{E}^{-}$. Now, we are prepared to formulate the main result on resonances.

Theorem 1.5. Let the self-adjoint operator $\mathrm{H}_{\varepsilon}, \varepsilon \in(0,1)$, be as in (1.5). Let the sheet $Z_{E} \subset \widetilde{Z}$ of the Riemann surface $\widehat{Z}$ be fixed. Define the associated set by:

$$
\mathcal{S}(E):=\left\{n \in \mathbb{N}:\left(l_{n-1}^{E}, l_{n}^{E}, l_{n+1}^{E}\right) \in\{(1,0,0),(0,1,1)\}\right\} .
$$

Let $\mathcal{R}_{E}(\varepsilon)$ be as in Definition 1.4 and set $\mathcal{R}_{E}^{-}(\varepsilon):=\mathcal{R}_{E}(\varepsilon) \cap \mathbb{C}_{-}$. Then, the following claims hold:

(i) $\mathcal{R}_{E}^{-}(\varepsilon) \subset \mathcal{U}(\varepsilon):=\left\{\lambda \in \mathbb{C}_{-}:\left|\nu_{n-1}-\lambda\right|\left|\nu_{n}-\lambda\right| \leq \varepsilon^{4} n^{2}, \forall n \in \mathbb{N}\right\}$.

(ii) For any $n \in \mathcal{S}(E)$ and sufficiently small $\varepsilon>0$ there is exactly one resonance $\lambda_{n}^{E}\left(\mathrm{H}_{\varepsilon}\right) \in \mathbb{C}_{-}$of $\mathrm{H}_{\varepsilon}$ on $Z_{E}^{-}$ lying in a neighbourhood of $\nu_{n}$, with the expansion

$$
\lambda_{n}^{E}\left(\mathrm{H}_{\varepsilon}\right)=\nu_{n}-\frac{\varepsilon^{4}}{16}[(2 n+1)+2 n(n+1) \mathrm{i}]+\mathcal{O}\left(\varepsilon^{5}\right), \quad \varepsilon \rightarrow 0+.
$$

(iii) For any $n \in \mathbb{N} \backslash \mathcal{S}(E)$ and all sufficiently small $\varepsilon, r>0$

$$
\mathcal{R}_{E}^{-}(\varepsilon) \cap \mathbb{D}_{r}\left(\nu_{n}\right)=\varnothing .
$$

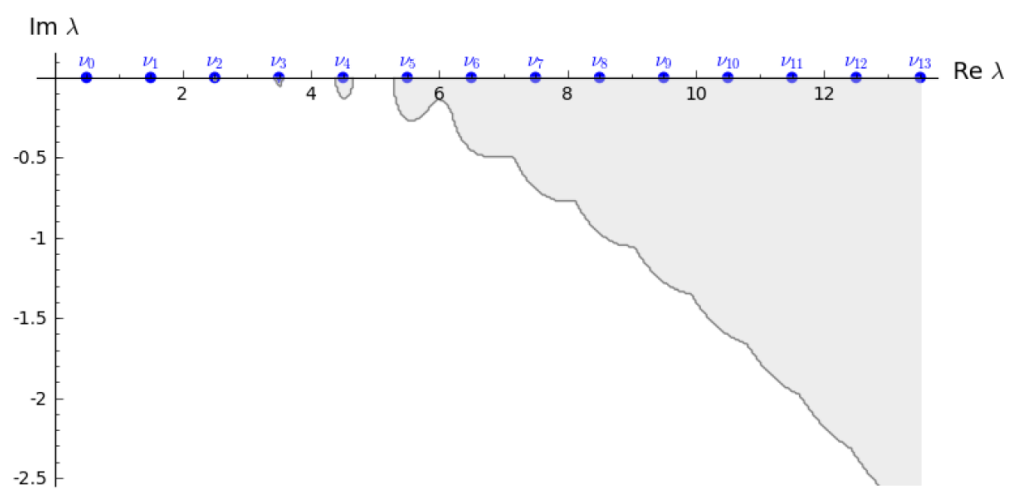

FIG. 1.1. The region $\mathcal{U}(0.12)$ (for $\varepsilon=0.12$ ) from Theorem 1.5 (i) (in grey) consists of 6 connected components. The components located in the neighbourhoods of the points $\nu_{0}, \nu_{1}, \nu_{2}$, $\nu_{3}$, are not visible because of being too small. The plot is performed with the aid of Sagemath.

In view of Theorem 1.5 (i) for sufficiently small $\varepsilon>0$, the resonances of $\mathrm{H}_{\varepsilon}$ on any sheet of $\widetilde{Z}$ are located in some vicinity of the thresholds $\nu_{n}$ (see Figure 1.1). Such behavior is typical for problems with many thresholds; see e.g. [11,13] and [14, $\S 2.4,3.4 .2]$. Note also that the estimate in Theorem 1.5 (i) reflects the correct order in $\varepsilon$ in the weak coupling limit $\varepsilon \rightarrow 0+$ given in Theorem 1.5 (ii). However, the coefficient of $\varepsilon^{4}$ in the definition of $\mathcal{U}(\varepsilon)$ can be probably improved. Observe also that $\mathcal{R}_{E}^{-}(\varepsilon) \subset \mathcal{U}(1)$ for any $\varepsilon \in(0,1)$.

According to Theorem 1.5 (ii)-(iii), the existence of a resonance near the threshold $\nu_{n}, n \in \mathbb{N}$, on a sheet $Z_{E}$ for small $\varepsilon>0$ depends only on the branches chosen for $r_{n-1}(\lambda), r_{n}(\lambda), r_{n+1}(\lambda)$ on $Z_{E}$. Although, one cannot exclude that higher order terms in the asymptotic expansion (1.14) depend on the branches chosen for other square roots. By exactly the same reason as in Theorem 1.2 (ii), we expect that the error term $\mathcal{O}\left(\varepsilon^{5}\right)$ in Theorem 1.5 (ii) can be replaced by $\mathcal{O}\left(\varepsilon^{6}\right)$. Theorem 1.5 (ii)-(iii) are proven by means of the Birman-Schwinger principle and the analytic implicit function theorem. The implicit scalar equation on resonances derived in Lemma 4.1 gives analyticity of $\varepsilon \mapsto \lambda_{n}^{E}\left(\mathrm{H}_{\varepsilon}\right)$ for small $\varepsilon>0$ and, as in the bound state case, it can be used to compute further terms in the expansion of $\lambda_{n}^{E}\left(\mathrm{H}_{\varepsilon}\right)$.

We point out that according to numerical tests that we performed, some resonances emerge from the inner points of the intervals $\left(\nu_{n}, \nu_{n+1}\right), n \in \mathbb{N}_{0}$, as $\varepsilon \rightarrow 1-$. The mechanism for the creation of these resonances is unclear at the moment.

Example 1.6. Let $E=\{1,2,4,5\}$. In this case $l^{E}=\{0,1,1,0,1,1,0,0,0,0,0, \ldots\}$ and we get that $\mathcal{S}(E)=$ $\{1,4,6\}$. By Theorem 1.5 (ii)-(iii) for all sufficiently small $\varepsilon>0$ there will be exactly one resonance on $Z_{E}^{-}$near $\nu_{1}, \nu_{4}, \nu_{6}$ and no resonances near the thresholds $\nu_{n}$ with $n \in \mathbb{N} \backslash\{1,4,6\}$. We confirm this result by numerical tests whose outcomes are shown in Figures 1.2 and 1.3. 

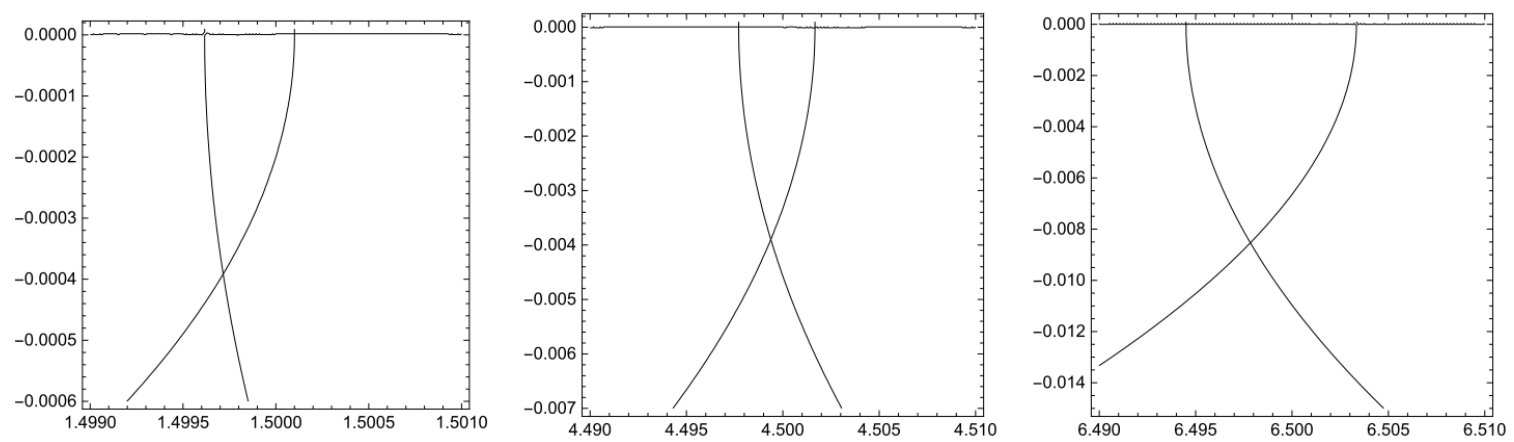

FIG. 1.2. Resonances of $H_{\varepsilon}$ with $\varepsilon=0.2$ lying on $Z_{E}^{-}$with $E=\{1,2,4,5\}$ are computed numerically with the help of Mathematica. Unique weakly coupled resonances near the thresholds $\nu_{1}=1.5, \nu_{4}=4.5, \nu_{6}=6.5$ are located at the intersections of the curves.

To plot Figure 1.2, we used the condition on resonances in Theorem 2.4 below. The infinite Jacobi matrix in this condition was truncated up to a reasonable finite size. Along the curves, respectively, the real and the imaginary part of the determinant of the truncated matrix vanishes. At the points of intersection of the curves the determinant itself vanishes. These points are expected to be close to true resonances ${ }^{3}$. We have also numerically verified that resonances do not exist near other low-lying thresholds $\nu_{n}$ with $n \in \mathbb{N} \backslash\{1,4,6\}$, which corresponds well to Theorem 1.5. In Figure 1.3 we summarize the results of all the numerical tests.

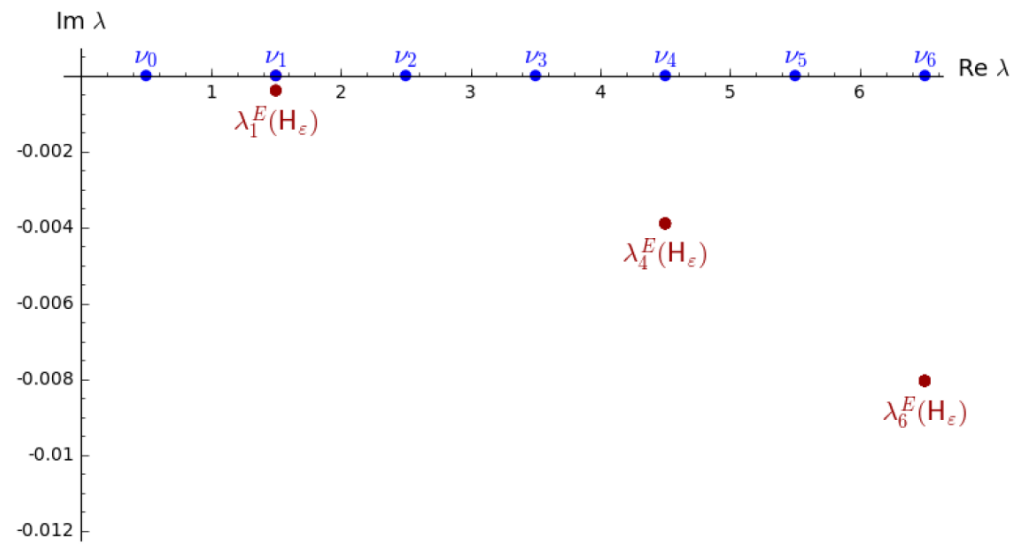

FIG. 1.3. Resonances of $\mathrm{H}_{\varepsilon}$ with $\varepsilon=0.2$ lying on $Z_{E}^{-}$with $E=\{1,2,4,5\}$.

Finally, we mention that no attempt has been made here to analyze the multiplicities of the resonances and to investigate resonances lying on $\widehat{Z} \backslash \widetilde{Z}$.

\section{Structure of the paper}

Birman-Schwinger-type principles for the characterization of eigenvalues and resonances of $H_{\varepsilon}$ are provided in Section 2. Theorem 1.2(i) on a lower bound for the first eigenvalue and Theorem 1.5 (i) on resonance free region are proven in Section 3. The aim of Section 4 is to prove Theorem 1.2 (ii) and Theorem 1.5 (ii)-(iii) on weakly coupled bound states and resonances. The proofs of technical statements formulated in Proposition 1.3 and Theorem 2.4 are postponed until Appendix.

\section{Birman-Schwinger-type conditions}

The Birman-Schwinger principle is a powerful tool for analyzing the discrete spectrum of a perturbed operator in the spectral gaps of the unperturbed one. This principle also has other various applications. Frequently, it can be generalized to detect resonances, defined as the poles of a meromorphic continuation of the (sandwiched)

\footnotetext{
${ }^{3}$ The analysis of convergence of the numerical method is beyond our scope.
} 
resolvent from the physical sheet to non-physical sheet(s) of the underlying Riemann surface. In the model under consideration, we encounter yet another manifestation of this principle.

In order to formulate a Birman-Schwinger-type condition on the bound states for $\mathrm{H}_{\varepsilon}$, we introduce the sequence of functions:

and the off-diagonal Jacobi matrix:

$$
b_{n}(\lambda):=\frac{n^{1 / 2}}{2\left(\nu_{n}-\lambda\right)^{1 / 4}\left(\nu_{n-1}-\lambda\right)^{1 / 4}}, \quad n \in \mathbb{N},
$$

$$
J(\lambda)=J\left(\{0\},\left\{b_{n}(\lambda)\right\}\right), \quad \lambda \in\left(0, \nu_{0}\right) .
$$

Recall that we use the same symbol $\mathrm{J}(\lambda)$ for the operator in $\ell^{2}\left(\mathbb{N}_{0}\right)$ generated by this matrix. It is straightforward to check that the operator $\mathrm{J}(\lambda)$ is bounded and self-adjoint. It can be easily verified that the difference $\mathrm{J}(\lambda)-\mathrm{J}_{0}$ is a compact operator. Therefore, one has $\sigma_{\text {ess }}(\mathrm{J}(\lambda))=\sigma_{\text {ess }}\left(\mathrm{J}_{0}\right)=[-1,1]$. Moreover, the operator $\mathrm{J}(\lambda)$ has simple eigenvalues $\pm \mu_{n}, \mu_{n}>1$, with the only possible accumulation points at $\mu= \pm 1$.

Theorem 2.1. [6, Thm. 3.1] Let the self-adjoint operator $\mathrm{H}_{\varepsilon}, \varepsilon \in(0,1)$, be as in (1.5) and let the Jacobi matrix $\mathrm{J}(\lambda)$ be as in (2.2). Then, the relation:

$$
\mathcal{N}\left((0, \lambda) ; \mathrm{H}_{\varepsilon}\right)=\mathcal{N}((1 / \varepsilon,+\infty) ; \mathrm{J}(\lambda)),
$$

holds for all $\lambda \in\left(0, \nu_{0}\right)$.

Remark 2.2. A careful inspection of the proof of [6, Thm 3.1] yields that Theorem 2.1 can also be modified, replacing (2.3) by:

$$
\mathcal{N}\left((0, \lambda] ; \mathrm{H}_{\varepsilon}\right)=\mathcal{N}([1 / \varepsilon,+\infty) ; \mathrm{J}(\lambda)) .
$$

In other words, the right endpoint of the interval $(0, \lambda)$ and the left endpoint of the interval $(1 / \varepsilon,+\infty)$ can be simultaneously included.

The following consequence of Theorem 2.1 and of the above remark will be useful further.

Corollary 2.3. Let the assumptions be as in Theorem 2.1. Then the following claims hold:

(i) $\varepsilon \mapsto \lambda_{k}\left(\mathrm{H}_{\varepsilon}\right)$ are continuous non-increasing functions;

(ii) $\operatorname{dim} \operatorname{ker}\left(\mathrm{H}_{\varepsilon}-\lambda\right)=\operatorname{dim} \operatorname{ker}(\mathrm{I}+\varepsilon \mathrm{J}(\lambda))$ for all $\lambda \in\left(0, \nu_{0}\right)$. In particular, since the eigenvalues of $\mathrm{J}(\lambda)$ are simple, the eigenvalues of $\mathrm{H}_{\varepsilon}$ are simple as well.

Proof. (i) Let $\varepsilon_{1} \in(0,1)$. For $\lambda=\lambda_{k}\left(\mathrm{H}_{\varepsilon_{1}}\right), k \in \mathbb{N}$, we have by Theorem 2.1 and Remark 2.2

$$
\mathcal{N}\left(\left[1 / \varepsilon_{1},+\infty\right) ; \mathrm{J}(\lambda)\right)=\mathcal{N}\left((0, \lambda] ; \mathrm{H}_{\varepsilon_{1}}\right) \geq k .
$$

Hence, for any $\varepsilon_{2} \in\left(\varepsilon_{1}, 1\right)$, we obtain:

$$
\mathcal{N}\left((0, \lambda] ; \mathrm{H}_{\varepsilon_{2}}\right)=\mathcal{N}\left(\left[1 / \varepsilon_{2},+\infty\right) ; \mathrm{J}(\lambda)\right) \geq \mathcal{N}\left(\left[1 / \varepsilon_{1},+\infty\right) ; \mathrm{J}(\lambda)\right) \geq k .
$$

Therefore, we get $\lambda_{k}\left(\mathrm{H}_{\varepsilon_{2}}\right) \leq \lambda=\lambda_{k}\left(\mathrm{H}_{\varepsilon_{1}}\right)$. Recall that $\mathrm{H}_{\varepsilon}$ represents the quadratic form $\mathfrak{h}_{\varepsilon}$ defined in (1.6). Continuity of the eigenvalues follows from [22, Thms. VI.3.6, VIII.1.14] and from the fact that the quadratic form:

$$
\operatorname{dom} \mathfrak{h}_{\varepsilon} \ni u \mapsto \varepsilon \sqrt{2}\left(\left.\operatorname{sign} y|y|^{1 / 2} u\right|_{\Sigma},\left.|y|^{1 / 2} u\right|_{\Sigma}\right)_{\mathbb{R}}, \quad \varepsilon \in(0,1),
$$

is relatively bounded with respect to

$$
\operatorname{dom} \mathfrak{h}_{\varepsilon} \ni u \mapsto\left\|\partial_{x} u\right\|_{\mathbb{R}^{2}}^{2}+\frac{1}{2}\left\|\partial_{y} u\right\|_{\mathbb{R}^{2}}^{2}+\frac{1}{2}(y u, y u)_{\mathbb{R}^{2}}
$$

with a bound less than one; $c f$. [6, Lem. 2.1].

(ii) By Theorem 2.1, Remark 2.2, and using symmetry of $\sigma(\mathrm{J}(\lambda))$ with respect to the origin we get:

$$
\begin{aligned}
\operatorname{dim} \operatorname{ker}\left(\mathrm{H}_{\varepsilon}-\lambda\right) & =\mathcal{N}\left((0, \lambda] ; \mathbf{H}_{\varepsilon}\right)-\mathcal{N}\left((0, \lambda) ; \mathbf{H}_{\varepsilon}\right) \\
& =\mathcal{N}([1 / \varepsilon,+\infty) ; \mathrm{J}(\lambda))-\mathcal{N}((1 / \varepsilon,+\infty) ; \mathrm{J}(\lambda))=\operatorname{dim} \operatorname{ker}(\mathrm{I}+\varepsilon \mathrm{J}(\lambda)) .
\end{aligned}
$$

For resonances of $H_{\varepsilon}$, one can derive a Birman-Schwinger-type condition analogous to the one in Corollary 2.3 (ii). For the sheet $Z_{E} \subset \widetilde{Z}$ of the Riemann surface $\widehat{Z}$, we define the Jacobi matrix:

$$
\mathrm{J}_{E}(\lambda):=\mathrm{J}\left(\{0\},\left\{b_{n}^{E}(\lambda)\right\}\right), \quad \lambda \in \mathbb{C} \backslash\left[\nu_{0},+\infty\right),
$$

where

$$
b_{n}^{E}(\lambda):=\frac{1}{2}\left(\frac{n}{r_{n}\left(\lambda, l_{n}^{E}\right) r_{n-1}\left(\lambda, l_{n-1}^{E}\right)}\right)^{1 / 2}, \quad n \in \mathbb{N} .
$$


The Jacobi matrix $J_{E}(\lambda)$ in (2.5) is closed, bounded, and everywhere defined in $\ell^{2}\left(\mathbb{N}_{0}\right)$, but in general nonselfadjoint. For $E=\varnothing$ and $\lambda \in\left(0, \nu_{0}\right)$ the Jacobi matrix $\mathrm{J}_{\varnothing}(\lambda)$ coincides with $\mathrm{J}(\lambda)$ in (2.2). In what follows it is also convenient to set $b_{0}^{E}(\lambda)=0$. In the next theorem, we characterize resonances of $\mathrm{H}_{\varepsilon}$ lying on the sheet $Z_{E}$.

Theorem 2.4. Let the self-adjoint operator $H_{\varepsilon}, \varepsilon \in(0,1)$, be as in (1.5). Let the sheet $Z_{E} \subset \widetilde{Z}$ be fixed, let $\mathcal{R}_{E}(\varepsilon)$ be as in Definition 1.4 and the associated operator-valued function $\mathrm{J}_{E}(\lambda)$ be as in (2.5). Then, the following equivalence holds:

$$
\lambda \in \mathcal{R}_{E}(\varepsilon) \quad \Longleftrightarrow \quad \operatorname{ker}\left(\mathrm{I}+\varepsilon \mathrm{J}_{E}(\lambda)\right) \neq\{0\} .
$$

For $E=\varnothing$, the claim of Theorem 2.4 follows from Corollary 2.3 (ii). The proof of the remaining part of Theorem 2.4 is postponed until Appendix. The argument essentially relies on Krein-type resolvent formula [7] for $\mathrm{H}_{\varepsilon}$ and on the analytic Fredholm theorem [25, Thm. 3.4.2].

Remark 2.5. Thanks to compactness of the difference $\mathrm{J}_{E}(\lambda)-\mathrm{J}_{0}$ we get by [23, Lem. XIII.4.3] that $\sigma_{\text {ess }}\left(\varepsilon \mathrm{J}_{E}(\lambda)\right)=$ $\left.\sigma_{\text {ess }}\left(\varepsilon J_{0}\right)\right)=[-\varepsilon, \varepsilon]$. Therefore, the equivalence (2.7) can be rewritten as:

$$
\lambda \in \mathcal{R}_{E}(\varepsilon) \quad \Longleftrightarrow \quad-1 \in \sigma_{\mathrm{d}}\left(\varepsilon \mathrm{J}_{E}(\lambda)\right)
$$

Identity $\mathrm{J}_{E}(\lambda)^{*}=\mathrm{J}_{E}(\bar{\lambda})$ combined with [22, Rem. III.6.23] and with Theorem 2.4 yields that the set $\mathcal{R}_{E}(\varepsilon)$ is symmetric with respect to the real axis.

\section{Localization of bound states and resonances}

In this section we prove Theorem 1.2 (i) and Theorem 1.5(i). The idea of the proof is to estimate the norm of $\mathrm{J}_{E}(\lambda)$ and to apply Corollary 2.3 (ii) and Theorem 2.4.

Proof of Theorem $1.2(i)$ and Theorem $1.5(i)$. The square of the norm of the operator $\mathrm{J}_{E}(\lambda)$ in $(2.5)$ can be estimated from above by:

$$
\begin{aligned}
\left\|\mathrm{J}_{E}(\lambda)\right\|^{2} & \leq \sup _{\xi \in \ell^{2}\left(\mathbb{N}_{0}\right),\|\xi\|=1}\left\|\mathrm{~J}_{E}(\lambda) \xi\right\|^{2} \leq \sup _{\xi \in \ell^{2}\left(\mathbb{N}_{0}\right),\|\xi\|=1}\left(\sum_{n \in \mathbb{N}_{0}}\left|b_{n}^{E}(\lambda) \xi_{n-1}+b_{n+1}^{E}(\lambda) \xi_{n+1}\right|^{2}\right) \\
& \leq \sup _{\xi \in \ell^{2}\left(\mathbb{N}_{0}\right),\|\xi\|=1}\left(2 \sum_{n \in \mathbb{N}_{0}}\left(\left|b_{n}^{E}(\lambda)\right|^{2}\left|\xi_{n-1}\right|^{2}+\left|b_{n+1}^{E}(\lambda)\right|^{2}\left|\xi_{n+1}\right|^{2}\right)\right) \\
& \leq 4 \sup _{n \in \mathbb{N}_{0}}\left|b_{n}^{E}(\lambda)\right|^{2} \sup _{\xi \in \ell^{2}\left(\mathbb{N}_{0}\right),\|\xi\|=1}\|\xi\|^{2}=4 \sup _{n \in \mathbb{N}}\left|b_{n}^{E}(\lambda)\right|^{2},
\end{aligned}
$$

where $b_{n}^{E}(\lambda), n \in \mathbb{N}_{0}$, are defined as in (2.6).

If $\left\|\varepsilon J_{E}(\lambda)\right\|<1$ holds for a point $\lambda \in \mathbb{C}_{-}$, then the condition $\operatorname{ker}\left(I+\varepsilon J_{E}(\lambda)\right) \neq\{0\}$ is not satisfied. Thus, $\lambda$ cannot by Theorem 2.4 be a resonance of $\mathrm{H}_{\varepsilon}$ lying on $Z_{E}^{-}$in the sense of Definition 1.4. In view of estimate (3.1) and of (2.6) to fulfil $\left\|\varepsilon J_{E}(\lambda)\right\|<1$, it suffices to satisfy:

or, equivalently,

$$
\frac{n}{\left|\nu_{n-1}-\lambda\right|^{1 / 2}\left|\nu_{n}-\lambda\right|^{1 / 2}}<\frac{1}{\varepsilon^{2}}, \quad \forall n \in \mathbb{N},
$$

$$
\left|\nu_{n}-\lambda\right| \cdot\left|\nu_{n-1}-\lambda\right|>\varepsilon^{4} n^{2}, \quad \forall n \in \mathbb{N} .
$$

Thus, the claim of Theorem 1.5 (i) is proven. If $\left\|\varepsilon J_{\varnothing}(\lambda)\right\|<1$ holds for a point $\lambda \in(0,1 / 2)$ then the condition $\operatorname{ker}\left(I+\varepsilon J_{\varnothing}(\lambda)\right) \neq\{0\}$ is not satisfied. Thus, by Corollary 2.3 (ii), $\lambda$ is not an eigenvalue of $\mathrm{H}_{\varepsilon}$. In view of (3.1) and (2.6) to fulfil $\left\|\varepsilon J_{\varnothing}(\lambda)\right\|<1$, it suffices to satisfy:

$$
\left(\nu_{n-1}-\lambda\right)\left(\nu_{n}-\lambda\right)=\lambda^{2}-2 n \lambda+n^{2}-1 / 4>n^{2} \varepsilon^{4}, \quad \forall n \in \mathbb{N} .
$$

The roots of the equation $\lambda^{2}-2 n \lambda+n^{2}-1 / 4-n^{2} \varepsilon^{4}=0$ are given by $\lambda_{n}^{ \pm}(\varepsilon)=n \pm \sqrt{1 / 4+n^{2} \varepsilon^{4}}$. Since $\lambda_{n}^{+}(\varepsilon)>1 / 2$ for all $n \in \mathbb{N}$, the condition (3.2) yields $\lambda_{1}\left(\mathrm{H}_{\varepsilon}\right) \geq \min _{n \in \mathbb{N}} \lambda_{n}^{-}(\varepsilon)$. For $n \in \mathbb{N}$ we have:

$$
\lambda_{n+1}^{-}(\varepsilon)-\lambda_{n}^{-}(\varepsilon)=1-\frac{(2 n+1) \varepsilon^{4}}{\left(\frac{1}{4}+n^{2} \varepsilon^{4}\right)^{1 / 2}+\left(\frac{1}{4}+(n+1)^{2} \varepsilon^{4}\right)^{1 / 2}} \geq 1-\frac{(2 n+1) \varepsilon^{4}}{(2 n+1) \varepsilon^{2}}=1-\varepsilon^{2}>0 .
$$

Hence, $\min _{n \in \mathbb{N}} \lambda_{n}^{-}(\varepsilon)=\lambda_{1}^{-}(\varepsilon)$ and the claim of Theorem 1.2 (i) follows. 


\section{The weak coupling regime: $\varepsilon \rightarrow 0+$}

In this section, we prove Theorem 1.2 (ii) and Theorem 1.5 (ii)-(iii). Intermediate results of this section given in Lemmata 4.1 and 4.3 are of an independent interest.

First, we introduce some auxiliary operators and functions. Let $n \in \mathbb{N}_{0}$ and the sheet $Z_{E} \subset \widetilde{Z}$ be fixed. We make use of notation $\mathrm{P}_{k l}:=\mathrm{e}_{n+k-2}\left(\cdot, \mathrm{e}_{n+l-2}\right)$ with $k, l \in\{1,2,3\}$. Note that for $n=0$ we have $\mathrm{P}_{k 1}=\mathrm{P}_{1 k}=0$ for $k=1,2,3$. It will also be convenient to decompose the Jacobi matrix $\mathrm{J}_{E}(\lambda)$ in (2.5) as:

$$
\mathrm{J}_{E}(\lambda)=\mathrm{S}_{n, E}(\lambda)+\mathrm{T}_{n, E}(\lambda)
$$

where the operator-valued functions $\lambda \mapsto \mathrm{T}_{n, E}(\lambda), \mathrm{S}_{n, E}(\lambda)$ are defined by:

$$
\mathrm{T}_{n, E}(\lambda):=b_{n+1}^{E}(\lambda)\left[\mathrm{P}_{23}+\mathrm{P}_{32}\right]+b_{n}^{E}(\lambda)\left[\mathrm{P}_{21}+\mathrm{P}_{12}\right], \quad \mathrm{S}_{n, E}(\lambda):=\mathrm{J}_{E}(\lambda)-\mathrm{T}_{n, E}(\lambda) .
$$

Clearly, the operator-valued function $\mathrm{S}_{n, E}(\cdot)$ is uniformly bounded on $\mathbb{D}_{1 / 2}\left(\nu_{n}\right)$. Moreover, for sufficiently small $r=r(n) \in(0,1 / 2)$ the bounded operator $\mathrm{I}+\varepsilon \mathrm{S}_{n, E}(\lambda)$ is at the same time boundedly invertible for all $(\varepsilon, \lambda) \in \Omega_{r}(n):=\mathbb{D}_{r} \times \mathbb{D}_{r}\left(\nu_{n}\right)$. Thus, the operator-valued function:

$$
\mathrm{R}_{n, E}(\varepsilon, \lambda):=\left(\mathrm{I}+\varepsilon \mathrm{S}_{n, E}(\lambda)\right)^{-1}
$$

is well-defined and analytic on $\Omega_{r}(n)$ and, in particular, $\mathrm{R}_{n, E}\left(0, \nu_{n}\right)=\mathrm{I}$. Furthermore, we introduce auxiliary scalar functions $\Omega_{r}(n) \ni(\varepsilon, \lambda) \mapsto f_{k l}^{E}(\varepsilon, \lambda)$ by:

$$
f_{k l}^{E}(\varepsilon, \lambda):=\left(\mathrm{R}_{n, E}(\varepsilon, \lambda) \mathrm{e}_{n+k-2}, \mathrm{e}_{n+l-2}\right), \quad k, l \in\{1,2,3\} .
$$

Thanks to $\mathrm{R}_{n, E}\left(0, \nu_{n}\right)=\mathrm{I}$ we have $f_{k l}^{E}\left(0, \nu_{n}\right)=\delta_{k l}$. Finally, we introduce $3 \times 3$ matrix-valued function:

$$
\mathbb{D}_{r} \times \mathbb{D}_{r}^{\times}\left(\nu_{n}\right) \ni(\varepsilon, \lambda) \mapsto \mathrm{A}_{n, E}(\varepsilon, \lambda):=\left(a_{k l}^{E}(\varepsilon, \lambda)\right)_{k, l=1}^{3,3}
$$

with the entries given for $k, l=1,2,3$ by:

$$
a_{k l}^{E}(\varepsilon, \lambda):=b_{n}^{E}(\lambda)\left(f_{1 k}^{E}(\varepsilon, \lambda) \delta_{2 l}+f_{2 k}^{E}(\varepsilon, \lambda) \delta_{1 l}\right)+b_{n+1}^{E}(\lambda)\left(f_{2 k}^{E}(\varepsilon, \lambda) \delta_{3 l}+f_{3 k}^{E}(\varepsilon, \lambda) \delta_{2 l}\right) .
$$

We remark that rank $\mathrm{A}_{n, E}(\varepsilon, \lambda) \leq 2$ due to linear dependence between the first and the third columns in $\mathrm{A}_{n, E}(\varepsilon, \lambda)$.

In the first lemma, we derive an implicit scalar equation which characterizes those points $\lambda \in \mathbb{C} \backslash\left[\nu_{0},+\infty\right)$ near $\nu_{n}$ for which the condition $\operatorname{ker}\left(I+\varepsilon J_{E}(\lambda)\right) \neq\{0\}$ is satisfied under additional assumption that $\varepsilon>0$ is small enough. This equation can be used to characterize the 'true' resonances for $H_{\varepsilon}$ as well as the weakly coupled bound state if $n=0$ and $E=\varnothing$.

Lemma 4.1. Let the self-adjoint operator $\mathrm{H}_{\varepsilon}, \varepsilon \in(0,1)$, be as in (1.5). Let $n \in \mathbb{N}_{0}$ and the sheet $Z_{E} \subset \widetilde{Z}$ be fixed. Let $r=r(n)>0$ be chosen as above. Then for all $\varepsilon \in(0, r)$ a point $\lambda \in \mathbb{D}_{r}\left(\nu_{n}\right) \backslash\left[\nu_{0}, \infty\right)$ is a resonance of $\mathrm{H}_{\varepsilon}$ on $Z_{E}$ if, and only if

$$
\operatorname{det}\left(\mathrm{I}+\varepsilon \mathrm{A}_{n, E}(\varepsilon, \lambda)\right)=0
$$

Proof. Using the decomposition (4.1) of $\mathrm{J}_{E}(\lambda)$ and the auxiliary operator in (4.3), we find:

$$
\operatorname{dim} \operatorname{ker}\left(I+\varepsilon \mathrm{J}_{E}(\lambda)\right)=\operatorname{dim} \operatorname{ker}\left(\mathrm{I}+\varepsilon \mathrm{S}_{n, E}(\lambda)+\varepsilon \mathrm{T}_{n, E}(\lambda)\right)=\operatorname{dim} \operatorname{ker}\left(\mathrm{I}+\varepsilon \mathrm{R}_{n, E}(\varepsilon, \lambda) \mathrm{T}_{n, E}(\lambda)\right) .
$$

Note that:

$$
\operatorname{rank}\left(\mathrm{R}_{n, E}(\varepsilon, \lambda) \mathrm{T}_{n, E}(\lambda)\right) \leq \operatorname{rank}\left(\mathrm{T}_{n, E}(\lambda)\right) \leq 3
$$

and, hence, using [26, Thm. $3.5(\mathrm{~b})]$, we get:

$$
\operatorname{dim} \operatorname{ker}\left(\mathrm{I}+\varepsilon \mathrm{R}_{n, E}(\varepsilon, \lambda) \mathrm{T}_{n, E}(\lambda)\right) \geq 1 \quad \Longleftrightarrow \quad \operatorname{det}\left(\mathrm{I}+\varepsilon \mathrm{R}_{n, E}(\varepsilon, \lambda) \mathrm{T}_{n, E}(\lambda)\right)=0 .
$$

For the orthogonal projector $\mathrm{P}:=\mathrm{P}_{11}+\mathrm{P}_{22}+\mathrm{P}_{33}$ the identity $\mathrm{T}_{n, E}(\lambda)=\mathrm{T}_{n, E}(\lambda) \mathrm{P}$ is straightforward. Hence, employing [27, IV.1.5] we find:

$$
\operatorname{det}\left(\mathrm{I}+\varepsilon \mathrm{R}_{n, E}(\varepsilon, \lambda) \mathrm{T}_{n, E}(\lambda)\right)=\operatorname{det}\left(\mathrm{I}+\varepsilon \mathrm{R}_{n, E}(\varepsilon, \lambda) \mathrm{T}_{n, E}(\lambda) \mathrm{P}\right)=\operatorname{det}\left(\mathrm{I}+\varepsilon \mathrm{PR}_{n, E}(\varepsilon, \lambda) \mathrm{T}_{n, E}(\lambda)\right) .
$$

For $k, l \in\{1,2,3\}$ we can write the following identities:

$$
\begin{aligned}
\mathrm{P}_{k k} \mathrm{PR}_{n, E}(\varepsilon, \lambda) \mathrm{T}_{n, E}(\lambda) \mathrm{P}_{l l} & =\mathrm{P}_{k k} \mathrm{R}_{n, E}(\varepsilon, \lambda)\left(b_{n}^{E}(\lambda)\left[\mathrm{P}_{21}+\mathrm{P}_{12}\right]+b_{n+1}^{E}(\lambda)\left[\mathrm{P}_{23}+\mathrm{P}_{32}\right]\right) \mathrm{P}_{l l} \\
& =\mathrm{P}_{k k} \mathrm{R}_{n, E}(\varepsilon, \lambda)\left(b_{n}^{E}(\lambda)\left[\mathrm{P}_{2 l} \delta_{1 l}+\mathrm{P}_{1 l} \delta_{2 l}\right]+b_{n+1}^{E}(\lambda)\left[\mathrm{P}_{2 l} \delta_{3 l}+\mathrm{P}_{3 l} \delta_{2 l}\right]\right) \\
& =\mathrm{P}_{k l} b_{n}^{E}(\lambda)\left[f_{2 k}^{E}(\varepsilon, \lambda) \delta_{1 l}+f_{1 k}^{E}(\varepsilon, \lambda) \delta_{2 l}\right]+\mathrm{P}_{k l} b_{n+1}^{E}(\lambda)\left[f_{2 k}^{E}(\varepsilon, \lambda) \delta_{3 l}+f_{3 k}^{E}(\varepsilon, \lambda) \delta_{2 l}\right] \\
& =a_{k l}^{E}(\varepsilon, \lambda) \mathrm{P}_{k l}
\end{aligned}
$$


with $f_{k l}^{E}$ as in (4.4), and as a result we get

$$
\mathrm{PR}_{n, E}(\varepsilon, \lambda) \mathrm{T}_{n, E}(\lambda)=\sum_{k=1}^{3} \sum_{l=1}^{3} a_{k l}^{E}(\varepsilon, \lambda) \mathrm{P}_{k l},
$$

with $a_{k l}^{E}(\varepsilon, \lambda)$ as in (4.6). Hence, the determinant in (4.9) can be expressed as:

$$
\operatorname{det}\left(\mathbf{I}+\varepsilon \mathrm{R}_{n, E}(\varepsilon, \lambda) \mathrm{T}_{n, E}(\lambda)\right)=\operatorname{det}\left(\mathbf{I}+\varepsilon \mathrm{A}_{n, E}(\varepsilon, \lambda)\right)
$$

where on the right-hand side we have the determinant of the $3 \times 3$ matrix $\mathrm{I}+\varepsilon \mathrm{A}_{n, E}(\varepsilon, \lambda) ; c f$. (4.5). The claim of lemma then follows from (4.7), (4.8), and Theorem 2.4.

In the second lemma, we establish the existence and investigate properties of solutions of the scalar equation in Lemma 4.1. To this aim it is natural to try to apply the analytic implicit function theorem. The main obstacle that makes a direct application of the implicit function theorem difficult lies in the fact that $\lambda \mapsto \operatorname{det}\left(I+\varepsilon \mathrm{A}_{n, E}(\varepsilon, \lambda)\right)$ is not analytic near $\nu_{n}$ due to the cut on the real axis. We circumvent this obstacle by applying the analytic implicit function theorem to an auxiliary function which is analytic in the disc and has values in different sectors of this disc that are in direct correspondence with the values of $\lambda \mapsto \operatorname{det}\left(I+\varepsilon \mathrm{A}_{n, E}(\varepsilon, \lambda)\right)$ on the four different sheets in $\widetilde{Z}$ which are mutually adjacent in a proper way.

Assumption 4.2. Let $n \in \mathbb{N}_{0}$ and the sheet $Z_{E} \subset \widetilde{Z}$ be fixed. Let the sheets $Z_{F}, Z_{G}$ and $Z_{H}$ be such that $Z_{E} \sim_{n-1} Z_{F}, Z_{F} \sim_{n} Z_{G}$ and $Z_{G} \sim_{n-1} Z_{H}$. For $r>0$ let the matrix-valued function $\mathbb{D}_{r} \times \mathbb{D}_{r}^{\times} \ni(\varepsilon, \kappa) \mapsto$ $\mathrm{B}_{n, E}(\varepsilon, \kappa)$ be defined by:

$$
\mathrm{B}_{n, E}(\varepsilon, \kappa):= \begin{cases}\mathrm{A}_{n, E}\left(\varepsilon, \nu_{n}-\kappa^{4}\right), & \arg \kappa \in \Phi_{E}:=\left(-\pi,-\frac{3 \pi}{4}\right] \cup\left(0, \frac{\pi}{4}\right], \\ \mathrm{A}_{n, F}\left(\varepsilon, \nu_{n}-\kappa^{4}\right), & \arg \kappa \in \Phi_{F}:=\left(-\frac{3 \pi}{4},-\frac{\pi}{2}\right] \cup\left(\frac{\pi}{4}, \frac{\pi}{2}\right], \\ \mathrm{A}_{n, G}\left(\varepsilon, \nu_{n}-\kappa^{4}\right), & \arg \kappa \in \Phi_{G}:=\left(-\frac{\pi}{2},-\frac{\pi}{4}\right] \cup\left(\frac{\pi}{2}, \frac{3 \pi}{4}\right], \\ \mathrm{A}_{n, H}\left(\varepsilon, \nu_{n}-\kappa^{4}\right), & \arg \kappa \in \Phi_{H}:=\left(-\frac{\pi}{4}, 0\right] \cup\left(\frac{3 \pi}{4}, \pi\right] .\end{cases}
$$

Tracing the changes in the characteristic vector along the path $Z_{E} \sim_{n-1} Z_{F} \sim_{n} Z_{G} \sim_{n-1} Z_{H}$, one easily verifies that $Z_{H} \sim_{n} Z_{E}$. Thus, $\mathrm{B}_{n, E}$ is analytic on $\mathbb{D}_{r} \times \mathbb{D}_{r}^{\times}$for sufficiently small $r>0$ which is essentially a consequence of componentwise analyticity in $\mathbb{D}_{r}$ of vector-valued function:

$$
\kappa \mapsto R_{\bullet}\left(\nu_{n}-\kappa^{4}\right), \quad \bullet \in\{E, F, G, H\} \quad \text { for } \quad \arg \kappa \in \Phi_{\bullet},
$$

where $R_{\bullet}$ is as in (1.9).

Lemma 4.3. Let $n \in \mathbb{N}_{0}$ and the sheet $Z_{E} \subset \widetilde{Z}$ be fixed. Set $(\mathfrak{p}, \mathfrak{q}, \mathfrak{r}):=\left(l_{n-1}^{E}, l_{n}^{E}, l_{n+1}^{E}\right)$. Let the matrix-valued function $\mathrm{B}_{n, E}$ be as in Assumption 4.2. Then the implicit scalar equation:

$$
\operatorname{det}\left(I+\varepsilon \mathrm{B}_{n, E}(\varepsilon, \kappa)\right)=0
$$

has exactly two solutions $\kappa_{n, E, j}(\cdot)$ analytic near $\varepsilon=0$ such that $\kappa_{n, E, j}(0)=0$, satisfying $\operatorname{det}\left(I+\varepsilon \mathrm{B}_{n, E}\left(\varepsilon, \kappa_{n, E, j}(\varepsilon)\right)\right)=0$ pointwise for sufficiently small $\varepsilon>0$, and having asymptotic expansions:

$$
\kappa_{n, E, j}(\varepsilon)=\varepsilon \frac{\left(z_{n, E}\right)_{j}^{1 / 2}}{2}+\mathcal{O}\left(\varepsilon^{2}\right), \quad \varepsilon \rightarrow 0+,
$$

where $z_{n, E}=(-1)^{\mathfrak{q}+\mathfrak{r}}(n+1)+(-1)^{\mathfrak{p}+\mathfrak{q}+1} n$ i.

Proof. First, we introduce the shorthand notations:

$$
u(\kappa):=b_{n}^{\bullet}\left(\nu_{n}-\kappa^{4}\right), \quad v(\kappa):=b_{n+1}^{\bullet}\left(\nu_{n}-\kappa^{4}\right), \quad \bullet \in\{E, F, G, H\} \quad \text { for } \quad \arg \kappa \in \Phi \bullet .
$$

Let $b_{k l}$ with $k, l \in\{1,2,3\}$ be the entries of the matrix-valued function $\mathrm{B}_{n, E}$. Furthermore, we define the scalar functions $X=X(\varepsilon, \kappa), Y=Y(\varepsilon, \kappa)$, and $Z=Z(\varepsilon, \kappa)$ by:

$$
\begin{aligned}
X & :=b_{11}+b_{22}+b_{33}, \\
Y & :=b_{11} b_{22}+b_{22} b_{33}+b_{11} b_{33}-b_{13} b_{31}-b_{12} b_{21}-b_{23} b_{32}, \\
Z & :=b_{11} b_{22} b_{33}+b_{13} b_{32} b_{21}+b_{12} b_{23} b_{31}-b_{13} b_{31} b_{22}-b_{12} b_{21} b_{33}-b_{11} b_{23} b_{32} .
\end{aligned}
$$

Employing an elementary formula for the determinant of $3 \times 3$ matrix, the equation $\operatorname{det}\left(\mathrm{I}+\varepsilon \mathrm{B}_{n, E}(\varepsilon, \kappa)\right)=0$ can be equivalently written as:

$$
1+\varepsilon X(\varepsilon, \kappa)+\varepsilon^{2} Y(\varepsilon, \kappa)+\varepsilon^{3} Z(\varepsilon, \kappa)=0 .
$$


By a purely algebraic argument, one can derive from (4.6) that $Z=0$. Hence, (4.12) simplifies to $1+\varepsilon X(\varepsilon, \kappa)+$ $\varepsilon^{2} Y(\varepsilon, \kappa)=0$. Introducing new parameter $t:=\varepsilon / \kappa$, we can further rewrite this equation as:

$$
1+t \kappa X(\varepsilon, \kappa)+t^{2} \kappa^{2} Y(\varepsilon, \kappa)=0 .
$$

Note also that the coefficients $(\varepsilon, \kappa) \mapsto \kappa X(\varepsilon, \kappa), \kappa^{2} Y(\varepsilon, \kappa)$ of the quadratic equation (4.13) are analytic in $\mathbb{D}_{r}^{2}$. For each fixed pair $(\varepsilon, \kappa)$ the equation (4.13) has (in general) two distinct roots $t_{j}(\varepsilon, \kappa), j=0,1$. The condition $\operatorname{det}\left(\mathrm{I}+\varepsilon \mathrm{B}_{n, E}(\varepsilon, \kappa)\right)=0$ with $\kappa \neq 0$ holds if, and only if at least one of the two conditions:

$$
f_{j}(\varepsilon, \kappa):=\varepsilon-\kappa t_{j}(\varepsilon, \kappa)=0, \quad j=0,1,
$$

is satisfied. Using analyticity of $u(\cdot)$ and $v(\cdot)$ near $\kappa=0$, we compute:

$$
\begin{aligned}
& \lim _{\kappa \rightarrow 0} \kappa u=\lim _{r \rightarrow 0+} r e^{\mathrm{i} \pi / 8} u\left(r e^{\mathrm{i} \pi / 8}\right)=\lim _{r \rightarrow 0+} \frac{n^{1 / 2}}{2} \frac{r e^{\mathrm{i} \pi / 8}}{\left(\left(-1+\mathrm{i} r^{4}\right)_{\mathfrak{p}}^{1 / 2}\left(\mathrm{i} r^{4}\right)_{\mathfrak{q}}^{1 / 2}\right)^{1 / 2}}=\frac{n^{1 / 2} e^{\mathrm{i} \pi / 8}}{2\left((-1)^{\left.\mathfrak{p}+\mathfrak{q} \mathrm{i} e^{\mathrm{i} \pi / 4}\right)^{1 / 2}},\right.} \\
& \lim _{\kappa \rightarrow 0} \kappa v=\lim _{r \rightarrow 0+} r e^{\mathrm{i} \pi / 8} v\left(r e^{\mathrm{i} \pi / 8}\right)=\lim _{r \rightarrow 0+} \frac{(n+1)^{1 / 2}}{2} \frac{r e^{\mathrm{i} \pi / 8}}{\left(\left(\mathrm{i} r^{4}\right)_{\mathfrak{q}}^{1 / 2}\left(1+\mathrm{i} r^{4}\right)_{\mathfrak{r}}^{1 / 2}\right)^{1 / 2}}=\frac{(n+1)^{1 / 2} e^{\mathrm{i} \pi / 8}}{2\left((-1)^{\mathfrak{q}+\mathfrak{r}} e^{\mathrm{i} \pi / 4}\right)^{1 / 2}} .
\end{aligned}
$$

Hence, we get:

$$
\begin{aligned}
\lim _{\varepsilon, r \rightarrow 0+} r e^{\mathrm{i} \pi / 8} b_{k l}\left(\varepsilon, r e^{\mathrm{i} \pi / 8}\right) & =\lim _{r \rightarrow 0+} r e^{\mathrm{i} \pi / 8} u\left(r e^{\mathrm{i} \pi / 8}\right)\left(f_{2 k}^{E}(\mathbf{0}) \delta_{3 l}+f_{3 k}^{E}(\mathbf{0}) \delta_{2 l}\right) \\
\quad+\lim _{r \rightarrow 0+} r e^{\mathrm{i} \pi / 8} v\left(r e^{\mathrm{i} \pi / 8}\right)\left(f_{1 k}^{E}(\mathbf{0}) \delta_{2 l}+f_{2 k}^{E}(\mathbf{0}) \delta_{1 l}\right) & \\
= & \frac{n^{1 / 2} e^{\mathrm{i} \pi / 8}\left(\delta_{2 k} \delta_{3 l}+\delta_{3 k} \delta_{2 l}\right)}{2\left((-1)^{\mathfrak{p}+\mathfrak{q}} \mathrm{i} e^{\mathrm{i} \pi / 4}\right)^{1 / 2}}+\frac{(n+1)^{1 / 2} e^{\mathrm{i} \pi / 8}\left(\delta_{1 k} \delta_{2 l}+\delta_{2 k} \delta_{1 l}\right)}{2\left((-1)^{\mathfrak{q}+\mathfrak{r}} e^{\mathrm{i} \pi / 4}\right)^{1 / 2}} .
\end{aligned}
$$

Combining this with (4.11) we end up with:

$$
\begin{aligned}
\lim _{(\varepsilon, \kappa) \rightarrow \mathbf{0}} \kappa X & =\lim _{\varepsilon, r \rightarrow 0+} r e^{\mathrm{i} \pi / 8} X\left(\varepsilon, r e^{\mathrm{i} \pi / 8}\right)=\lim _{\varepsilon, r \rightarrow 0+} r e^{\mathrm{i} \pi / 8}\left[b_{11}+b_{22}+b_{33}\right]\left(\varepsilon, r e^{\mathrm{i} \pi / 8}\right)=0 \\
\lim _{(\varepsilon, \kappa) \mathbf{0}} \kappa^{2} Y & =\lim _{\varepsilon, r \rightarrow 0+} r^{2} e^{\mathrm{i} \pi / 4} Y\left(\varepsilon, r e^{\mathrm{i} \pi / 8}\right) \\
& =\lim _{\varepsilon, r \rightarrow 0+} r^{2} e^{\mathrm{i} \pi / 4}\left[b_{11} b_{22}+b_{22} b_{33}+b_{11} b_{33}-b_{13} b_{31}-b_{12} b_{21}-b_{23} b_{32}\right]\left(\varepsilon, r e^{\mathrm{i} \pi / 8}\right) \\
& =\lim _{\varepsilon, r \rightarrow 0+} r^{2} e^{\mathrm{i} \pi / 4}\left[-b_{12} b_{21}-b_{23} b_{32}\right]\left(\varepsilon, r e^{\mathrm{i} \pi / 8}\right) \\
& =-\left(\frac{n^{1 / 2} e^{\mathrm{i} \pi / 8}}{2\left((-1)^{\mathfrak{p}+\mathfrak{q}} \mathrm{i} e^{\mathrm{i} \pi / 4}\right)^{1 / 2}}\right)^{2}-\left(\frac{(n+1)^{1 / 2} e^{\mathrm{i} \pi / 8}}{2\left((-1)^{\mathfrak{q}+\mathfrak{r}} e^{\mathrm{i} \pi / 4}\right)^{1 / 2}}\right)^{2} \\
& =-\frac{(-1)^{\mathfrak{p}+\mathfrak{q}+1} n \mathrm{i}}{4}-\frac{(-1)^{\mathfrak{q}+\mathfrak{r}}(n+1)}{4}=-\frac{z_{n, E}}{4} .
\end{aligned}
$$

Hence, the roots $t_{j}(\varepsilon, \kappa)$ of (4.13) converge in the limit $(\varepsilon, \kappa) \rightarrow \mathbf{0}$ to the roots $2\left[\left(z_{n, E}\right)_{j}^{1 / 2}\right]^{-1}, j=0,1$, of the quadratic equation $z_{n, E} t^{2}-4=0$. Moreover, analyticity of the coefficients in equation (4.13), the above limits, and the formula for the roots of a quadratic equation imply analyticity of the functions $(\varepsilon, \kappa) \mapsto t_{j}(\varepsilon, \kappa)$ near $\mathbf{0}$.

Step 2. The partial derivatives of $f_{j}$ in (4.14) with respect to $\varepsilon$ and $\kappa$ are given by $\partial_{\varepsilon} f_{j}=1-\kappa \partial_{\varepsilon} t_{j}$ and $\partial_{\kappa} f_{j}=-t_{j}-\kappa \partial_{\kappa} t_{j}$. Analyticity of $t_{j}$ near $\mathbf{0}$ implies $\left(\partial_{\varepsilon} f_{j}\right)(\mathbf{0})=1$ and $\left(\partial_{\kappa} f_{j}\right)(\mathbf{0})=-t_{j}$. In particular, we have shown that $\left(\partial_{\kappa} f_{j}\right)(\mathbf{0}) \neq 0$. Since the functions $f_{j}(\cdot)$ are analytic near $\mathbf{0}$ and satisfy $f_{j}(\mathbf{0})=0$, we can apply the analytic implicit function theorem [25, Thm. 3.4.2] which yields existence of a unique function $\kappa_{j}(\cdot)$, analytic near $\varepsilon=0$ such that $\kappa_{j}(0)=0$ and that $f_{j}\left(\varepsilon, \kappa_{j}(\varepsilon)\right)=0$ holds pointwise. Moreover, the derivative of $\kappa_{j}$ at $\varepsilon=0$ can be expressed as:

$$
\kappa_{j}^{\prime}(0)=-\frac{\left(\partial_{\varepsilon} f_{j}\right)(\mathbf{0})}{\left(\partial_{\kappa} f_{j}\right)(\mathbf{0})}=\frac{1}{t_{j}(\mathbf{0})} .
$$

Hence, we obtain Taylor expansion for $\kappa_{j}$ near $\varepsilon=0$ :

$$
\kappa_{j}(\varepsilon)=\kappa_{j}(0)+\kappa_{j}^{\prime}(0) \varepsilon+\mathcal{O}\left(\varepsilon^{2}\right)=\frac{\varepsilon}{t_{j}(\mathbf{0})}+\mathcal{O}\left(\varepsilon^{2}\right)=\varepsilon \frac{\left(z_{n, E}\right)_{j}^{1 / 2}}{2}+\mathcal{O}\left(\varepsilon^{2}\right) \quad \varepsilon \rightarrow 0+.
$$

The functions $\kappa_{j}, j=0,1$, satisfy all the requirements in the claim of the lemma.

Now we are prepared to prove Theorem 1.2(ii) and Theorem 1.5(ii)-(iii) from the introduction. 
Proof of Theorem 1.2 (ii). By Proposition 1.1 (iv) we have $\mathcal{N}_{1 / 2}\left(\mathrm{H}_{\varepsilon}\right)=1$ for all sufficiently small $\varepsilon>0$. Recall that we denote by $\lambda_{1}\left(\mathrm{H}_{\varepsilon}\right)$ the corresponding unique eigenvalue. Thus, we have by Lemma 4.1:

$$
\operatorname{det}\left(\mathrm{I}+\varepsilon \mathrm{A}_{0, \varnothing}\left(\varepsilon, \lambda_{1}\left(\mathrm{H}_{\varepsilon}\right)\right)\right)=0 .
$$

Using the construction of Assumption 4.2 for the physical sheet and $n=0$, we obtain

$$
\operatorname{det}\left(\mathrm{I}+\varepsilon \mathrm{B}_{0, \varnothing}\left(\varepsilon,\left(\nu_{0}-\lambda_{1}\left(\mathrm{H}_{\varepsilon}\right)\right)^{1 / 4}\right)\right)=\operatorname{det}\left(\mathrm{I}+\varepsilon \mathrm{A}_{0, \varnothing}\left(\varepsilon, \lambda_{1}\left(\mathrm{H}_{\varepsilon}\right)\right)\right)=0,
$$

where we have chosen the principal branch for $(\cdot)^{1 / 4}$. Thus, by Lemma 4.3 , we get:

$$
\left(\nu_{0}-\lambda_{1}\left(\mathrm{H}_{\varepsilon}\right)\right)^{1 / 4}=\frac{\varepsilon}{2}+\mathcal{O}\left(\varepsilon^{2}\right), \quad \varepsilon \rightarrow 0+,
$$

where we have used the fact that $z_{0, \varnothing}=1$. Hence, taking the fourth power of the left and right hand sides in the above equation we arrive at:

$$
\lambda_{1}\left(\mathrm{H}_{\varepsilon}\right)=\nu_{0}-\frac{\varepsilon^{4}}{16}+\mathcal{O}\left(\varepsilon^{5}\right), \quad \varepsilon \rightarrow 0+
$$

Proof of Theorem 1.5 (ii)-(iii). Let $n \in \mathbb{N}$ and the sheet $Z_{E} \subset \widetilde{Z}$ be fixed. Let us repeat the construction of Assumption 4.2. By Lemma 4.3 we infer that there exist exactly two analytic solutions $\kappa_{n, E, j}, j=0,1$ of the implicit scalar equation $\operatorname{det}\left(\mathrm{I}+\varepsilon \mathrm{B}_{n, E}(\varepsilon, \kappa)\right)=0$ such that $\kappa_{n, E, j}(0)=0$. It can be checked that both solutions correspond to the same resonance and it suffices to analyze the solution $\kappa_{n, E}:=\kappa_{n, E, 0}$ only.

For all small enough $\varepsilon>0$ the asymptotics (4.10) yields:

$$
\arg \left(\kappa_{n, E}(\varepsilon)\right)=\frac{1}{2} \arg \left(z_{n, E}\right) \in \Phi_{E}, \quad \text { if, and only if } n \in \mathcal{S}(E) .
$$

Hence, if $n \in \mathbb{N} \backslash \mathcal{S}(E)$, Lemmata 4.1 and 4.3 imply that there will be no resonances in the vicinity of the point $\lambda=\nu_{n}$ lying on $Z_{E}^{-}$for sufficiently small $\varepsilon>0$. Thus, we have proven Theorem 1.5 (iii). While if $n \in \mathcal{S}(E)$ we get by Lemmata 4.1 and 4.3 that there will be exactly one resonance

$$
\lambda_{n}^{E}\left(\mathrm{H}_{\varepsilon}\right)=\nu_{n}-\left(\kappa_{n, E}(\varepsilon)\right)^{4},
$$

in the vicinity of the point $\lambda=\nu_{n}$ lying on $Z_{E}^{-}$for sufficiently small $\varepsilon>0$ and its asymptotic expansion is a direct consequence of the asymptotic expansion (4.10) given in Lemma 4.3. Thus, the claim of Theorem 1.5 (ii) follows.

\section{APPENDIX}

\section{A. Krein's formula, meromorphic continuation of resolvent, and condition on resonances}

In this appendix, we use Krein's resolvent formula for Smilansky Hamiltonian to prove Proposition 1.3 and Theorem 2.4 on meromorphic continuation of $\left(\mathrm{H}_{\varepsilon}-\lambda\right)^{-1}$ to $\widetilde{Z}$. The proposed continuation procedure is of an iterative nature wherein, we first extend $\left(\mathrm{H}_{\varepsilon}-\lambda\right)^{-1}$ to the sheets adjacent to the physical sheet, then to the sheets which are adjacent to the sheets being adjacent to the physical sheet and so on. by:

To this aim, we define for $n \in \mathbb{N}_{0}$ the scalar functions $\mathbb{C} \backslash\left[\nu_{0},+\infty\right) \mapsto y_{n}(\lambda)$ and $\left(\mathbb{C} \backslash\left[\nu_{0},+\infty\right)\right) \times \mathbb{R} \mapsto \eta_{n}(\lambda ; x)$

$$
y_{n}(\lambda):=r_{n}(\lambda) \sqrt{\nu_{n}}, \quad \eta_{n}(\lambda ; x):=\nu_{n}^{1 / 4} \exp \left(-r_{n}(\lambda)|x|\right)
$$

where $r_{n}(\cdot), n \in \mathbb{N}_{0}$, is as in (1.7). Next, we introduce the following operator-valued function:

$$
\mathrm{T}(\lambda): \ell^{2}\left(\mathbb{N}_{0}\right) \rightarrow \mathcal{H}, \quad \mathrm{T}(\lambda)\left\{c_{n}\right\}:=\left\{c_{n} \eta_{n}(\lambda ; x)\right\}
$$

For each fixed $\lambda \in \mathbb{C} \backslash\left[\nu_{0},+\infty\right)$ the operator $\mathrm{T}(\lambda)$ is bounded and everywhere defined and the adjoint of $\mathrm{T}(\bar{\lambda})$ acts as:

$$
\mathrm{T}(\bar{\lambda})^{*}\left\{u_{n}\right\} \sim\left\{I_{n}\left(\lambda ; u_{n}\right)\right\}_{n \in \mathbb{N}_{0}}, \quad I_{n}(\lambda ; u):=\int_{\mathbb{R}} \eta_{n}(\lambda ; x) u(x) \mathrm{d} x .
$$

With these preparations, the resolvent difference of $\mathrm{H}_{\varepsilon}$ and $\mathrm{H}_{0}$ can be expressed by [7, Thm. 6.1] (see also [4, Sec. 6]) as follows:

$$
\left(\mathrm{H}_{\varepsilon}-\lambda\right)^{-1}=\left(\mathrm{H}_{0}-\lambda\right)^{-1}+\mathrm{T}(\lambda) \mathrm{Y}(\lambda)\left[\left(\mathrm{I}+\varepsilon \mathrm{J}_{\varnothing}(\lambda)\right)^{-1}-\mathrm{I}\right] \mathrm{Y}(\lambda) \mathrm{T}(\bar{\lambda})^{*}, \quad \lambda \in \mathbb{C} \backslash\left[\nu_{0},+\infty\right),
$$

where $\mathrm{H}_{0}$ is the Smilansky Hamiltonian with $\varepsilon=0, \mathrm{Y}(\lambda)=\operatorname{diag}\left\{\left(2 y_{n}(\lambda)\right)^{-1 / 2}\right\}$ and $\mathrm{J}_{\varnothing}(\lambda)$ is as in (2.5). The formula (A.2) can be viewed as a particular case of abstract Krein's formula (see e.g. [29-31]) for the resolvent difference of two self-adjoint extensions of their common densely defined symmetric restriction. 
Proof of Proposition 1.3 and Theorem 2.4. Let us fix $n \in \mathbb{N}_{0}$ and a sheet $Z_{E} \subset \widetilde{Z}$. We denote by $\mathrm{R}_{n}(\lambda)$ the resolvent of the self-adjoint operator $H^{2}(\mathbb{R}) \ni f \mapsto-f^{\prime \prime}+\nu_{n} f$ in the Hilbert space $L^{2}(\mathbb{R})$. We can express the function $\mathfrak{r}_{n, \varepsilon}^{\varnothing}(\cdot ; u)$ in (1.13) using Krein's formula (A.2) as:

$$
\begin{aligned}
\mathfrak{r}_{n, \varepsilon}^{\varnothing}(\lambda ; u) & =\left\langle\left(\mathrm{H}_{\varepsilon}-\lambda\right)^{-1} u \otimes \mathrm{e}_{n}, u \otimes \mathrm{e}_{n}\right\rangle \\
& =\left\langle\left(\mathrm{H}_{0}-\lambda\right)^{-1} u \otimes \mathrm{e}_{n}, u \otimes \mathrm{e}_{n}\right\rangle+\left(\mathrm{Y}(\lambda)\left[\left(\mathrm{I}+\varepsilon \mathrm{J}_{\varnothing}(\lambda)\right)^{-1}-\mathrm{I}\right] \mathrm{Y}(\lambda) \mathrm{T}(\bar{\lambda})^{*} u \otimes \mathrm{e}_{n}, \mathrm{~T}(\lambda)^{*} u \otimes \mathrm{e}_{n}\right) \\
& =\left(\mathrm{R}_{n}(\lambda) u, u\right)_{\mathbb{R}}+I_{n}(\lambda ; u) \overline{I_{n}(\bar{\lambda} ; u)}\left(\left[\left(\mathrm{I}+\varepsilon \mathrm{J}_{\varnothing}(\lambda)\right)^{-1}-\mathrm{I}\right] \mathrm{Y}(\lambda) \mathrm{e}_{n}, \mathrm{Y}(\lambda)^{*} \mathrm{e}_{n}\right) \\
& =\left(\mathrm{R}_{n}(\lambda) u, u\right)_{\mathbb{R}}+\frac{I_{n}(\lambda ; u) I_{n}(\lambda ; \bar{u})}{2 y_{n}(\lambda)}\left[\left(\left(\mathrm{I}+\varepsilon \mathrm{J}_{\varnothing}(\lambda)\right)^{-1} \mathrm{e}_{n}, \mathrm{e}_{n}\right)-1\right] .
\end{aligned}
$$

Since $\left(\mathrm{R}_{n}(\lambda) u, u\right)_{\mathbb{R}}, y_{n}(\lambda), I_{n}(\lambda ; u)$, and $I_{n}(\lambda ; \bar{u})$ can be easily analytically continued to $\widetilde{Z}$, to extend $\mathfrak{r}_{n, \varepsilon}^{\varnothing}(\cdot ; u)$ meromorphically to the other sheets of the component $\widetilde{Z}$ it suffices to extend:

$$
\mathfrak{s}_{n, \varepsilon}^{\varnothing}(\lambda):=\left(\left(\mathrm{I}+\varepsilon \mathrm{J}_{\varnothing}(\lambda)\right)^{-1} \mathrm{e}_{n}, \mathrm{e}_{n}\right)
$$

meromorphically from $Z_{\varnothing}$ to $\widetilde{Z}$. The poles of the meromorphic extension of $\mathfrak{s}_{n, \varepsilon}^{\varnothing}(\cdot)$ can be identified with the resonances of $\mathrm{H}_{\varepsilon}$ in the sense of Definition 1.4.

To this aim, we set by definition:

$$
\mathfrak{s}_{n, \varepsilon}^{E}(\lambda):=\left(\left(\mathrm{I}+\varepsilon \mathrm{J}_{E}(\lambda)\right)^{-1} \mathrm{e}_{n}, \mathrm{e}_{n}\right)
$$

for any $\lambda \in \mathbb{C} \backslash\left[\nu_{0},+\infty\right)$ such that $-1 \notin \sigma\left(\varepsilon J_{E}(\lambda)\right)$. In what follows, we let $Z_{E}$ and $Z_{F}$ be two sheets of $\widetilde{Z}$ such that $Z_{E} \sim_{n-1} Z_{F}$ with $n \in \mathbb{N}_{0}{ }^{4}$. Suppose that $\lambda \mapsto \mathfrak{s}_{n, \varepsilon}^{E}(\cdot)$ is well defined and meromorphic either on $Z_{E}^{+}$or on $Z_{E}^{-}$. Next, we extend $\lambda \mapsto \mathfrak{s}_{n, \varepsilon}^{E}(\cdot)$ meromorphically from $Z_{E}^{ \pm}$to $Z_{F}^{\mp}$. Without loss of generality, we restrict our attention to the case that $\lambda \mapsto \mathfrak{s}_{n, \varepsilon}^{E}(\cdot)$ is meromorphic on $Z_{E}^{+}$and extend it meromorphically to $Z_{F}^{-}$. On the open set $\Omega_{n}:=\mathbb{C}_{+} \cup \mathbb{C}_{-} \cup\left(\nu_{n-1}, \nu_{n}\right)$, the operator-valued function:

$$
\mathrm{J}_{E F}(\lambda):= \begin{cases}\mathrm{J}_{E}(\lambda), & \lambda \in \mathbb{C}_{+}, \\ \mathrm{J}_{F}(\lambda), & \lambda \in \Omega_{n} \backslash \mathbb{C}_{+},\end{cases}
$$

is analytic which is essentially a consequence of analyticity on $\Omega_{n}$ of the entries $b_{m}^{\bullet}(\lambda)$ (with $\bullet=E$ for $\lambda \in \mathbb{C}_{+}$ and $\bullet=F$ for $\lambda \in \mathbb{C}_{-}$) for the underlying Jacobi matrix. Thus, the operator-valued function:

$$
\Omega_{n} \ni \lambda \mapsto \mathrm{A}_{\varepsilon}^{E F}(\lambda):=\varepsilon\left(\mathrm{I}+\varepsilon \mathrm{J}_{0}\right)^{-1}\left(\mathrm{~J}_{E F}(\lambda)-\mathrm{J}_{0}\right)
$$

is also analytic on $\Omega_{n}$ because of the analyticity of $\mathrm{J}_{E F}(\lambda)$. Furthermore, the values of $\mathrm{A}_{\varepsilon}^{E F}(\cdot)$ are compact operators thanks to compactness of the difference $\mathrm{J}_{E F}(\lambda)-\mathrm{J}_{0}$. Taking into account that:

$$
\left(\left(\mathrm{I}+\mathrm{A}_{\varepsilon}^{E F}(\lambda)\right)^{-1} \mathrm{e}_{n},\left(\mathrm{I}+\varepsilon \mathrm{J}_{0}\right)^{-1} \mathrm{e}_{n}\right)= \begin{cases}\mathfrak{s}_{n, \varepsilon}^{E}(\lambda), & \lambda \in \mathbb{C}_{+}, \\ \mathfrak{s}_{n, \varepsilon}^{F}(\lambda), & \lambda \in \Omega_{n} \backslash \mathbb{C}_{+},\end{cases}
$$

we obtain from the analytic Fredholm theorem [28, Thm. VI.14] that $\mathbb{C}_{-} \ni \lambda \mapsto \mathfrak{s}_{n, \varepsilon}^{F}(\lambda)$ is a meromorphic continuation of $\mathbb{C}_{+} \ni \lambda \mapsto \mathfrak{s}_{n, \varepsilon}^{E}(\lambda)$ across the interval $\left(\nu_{n-1}, \nu_{n}\right)$ and that the poles of $\mathbb{C}_{-} \ni \lambda \mapsto \mathfrak{s}_{n, \varepsilon}^{F}(\lambda)$ satisfy the condition:

$$
\operatorname{ker}\left(I+\varepsilon J_{F}(\lambda)\right) \neq\{0\}, \quad \lambda \in \mathbb{C}_{-} .
$$

Starting from the physical sheet $Z_{\varnothing}$, we use the above procedure iteratively to extend $\mathfrak{s}_{n, \varepsilon}^{\varnothing}(\cdot)$ meromorphically to the whole of $\widetilde{Z}$ thus proving Proposition 1.3 and Theorem 2.4.

\section{Acknowledgements}

This research was supported by the Czech Science Foundation (GAČR) within the project 14-06818S.

\footnotetext{
${ }^{4}$ Recall that for any sheet $Z_{E}$ holds $Z_{E} \sim_{-1} Z_{E}$.
} 


\section{References}

[1] Smilansky U. Irreversible quantum graphs. Waves Random Media, 2004, 14 (1), S143-S153.

[2] Evans W.D., Solomyak M. Smilansky's model of irreversible quantum graphs. I: The absolutely continuous spectrum. J. Phys. A: Math. Gen., 2005, 38 (21), P. 4611-4627.

[3] Evans W. D., Solomyak M. Smilansky's model of irreversible quantum graphs. II: The point spectrum. J. Phys. A: Math. Gen., 2005, 38 (35), P. 7661-7675.

[4] Naboko S., Solomyak M. On the absolutely continuous spectrum in a model of an irreversible quantum graph. Proc. Lond. Math. Soc. (3), 2006, 92 (1), P. 251-272.

[5] Rozenblum G., Solomyak M. On a family of differential operators with the coupling parameter in the boundary condition. J. Comput. Appl. Math., 2007, 208 (1), P. 57-71.

[6] Solomyak M. On the discrete spectrum of a family of differential operators. Funct. Anal. Appl., 2004,38 (3), P. $217-223$.

[7] Solomyak M. On a mathematical model of irreversible quantum graphs. St. Petersburg Math. J., 2006,17 (5), P. 835-864.

[8] Solomyak M. On the limiting behaviour of the spectra of a family of differential operators. J. Phys. A: Math. Gen., 2006, 39 (33), P. 10477-10489.

[9] Guarneri I. Irreversible behaviour and collapse of wave packets in a quantum system with point interactions. J. Phys. A: Math. Theor. 2011, 44 (48), 485304, 22 p.

[10] Aslanyan A., Parnovski L., and Vassiliev D. Complex resonances in acoustic waveguides. Q. J. Mech. Appl. Math., 2000, 53 (3), P. 429-447.

[11] Duclos P., Exner P., Meller B. Open quantum dots: Resonances from perturbed symmetry and bound states in strong magnetic fields. Rep. Math. Phys., 2001, 47 (2), P. 253-267.

[12] Edward J. On the resonances of the Laplacian on waveguides. J. Math. Anal. Appl., 2002, 272 (1), P. 89-116.

[13] Exner P., Gawlista R., Šeba P., Tater M. Point interactions in a strip. Ann. Phys., 1996, 252 (1), P. $133-179$.

[14] Exner P., Kovařík H. Quantum waveguides. Springer, Cham., 2015, 382 p.

[15] Christiansen T. Some upper bounds on the number of resonances for manifolds with infinite cylindrical ends. Ann. Henri Poincaré, 2002, 3 (5), P. 895-920.

[16] Christiansen T. Asymptotics for a resonance-counting function for potential scattering on cylinders. J. Funct. Anal., 2004, 216 (1), P. $172-190$.

[17] Barseghyan D., Exner P. Spectral estimates for a class of Schrödinger operators with infinite phase space and potential unbounded from below. J. Phys. A: Math. Theor, 2012, 45 (7), 075204, 14 p.

[18] Barseghyan D., Exner P. A regular version of Smilansky model. J. Math. Phys., 2014, 55 (4), 042104,13 p.

[19] Barseghyan D., Exner P., Khrabustovskyi A., and Tater M. Spectral analysis of a class of Schrödinger operators exhibiting a parameterdependent spectral transition. J. Phys. A: Math. Theor., 2016, 49 (16), 165302.

[20] Abramowitz M., Stegun I. Handbook of Mathematical Functions with Formulas, Graphs, and Mathematical Tables, U.S. Government Printing Office, Washington, D.C:, 1964. 1046 p.

[21] Chihara T. An introduction to orthogonal polynomials. Gordon and Breach Science Publishers, New York-London-Paris., 1978,249 p.

[22] Kato T. Perturbation theory for linear operators. Springer-Verlag, Berlin, 1995, 619 p.

[23] Reed M., Simon B. Methods of modern mathematical physics. IV. Analysis of operators. Academic Press, New York, 1978,396 p.

[24] Motovilov A. Analytic continuation of $S$ matrix in multichannel problems. Theoret. Math. Phys., 1993,95 (3), P. $692-699$.

[25] Simon B. Basic complex analysis. A comprehensive course in analysis, part 2A. American Mathematical Society, Providence, 2015 , 641 p.

[26] Simon B. Trace ideals and their applications. 2nd ed. American Mathematical Society, Providence, $2005,150 \mathrm{p}$.

[27] Gohberg I. C., Krein M. G. Introduction to the theory of linear nonselfadjoint operators. Translations of Mathematical Monographs, American Mathematical Society, Providence, RI, 1969, 378 p.

[28] Reed M., Simon B. Methods of modern mathematical physics. I: Functional analysis. Academic Press, New York, 1980,400 p.

[29] Behrndt J., Langer M. Elliptic operators, Dirichlet-to-Neumann maps and quasi boundary triples. in: Operator Methods for Boundary Value Problems, London Math. Soc. Lecture Note Series, 2012, 404, P. 121-160.

[30] Brüning J., Geyler V., Pankrashkin K. Spectra of self-adjoint extensions and applications to solvable Schrödinger operators. Rev. Math. Phys., 2008, 20 (1), P. 1-70.

[31] Derkach V., Malamud M. Generalized resolvents and the boundary value problems for Hermitian operators with gaps. J. Funct. Anal., 1991, 95 (1), P. 1-95. 